\title{
MFG-E8 attenuates neuro-inflammation in subarachnoid hemorrhage through driving microglial M2 polarization via modulating Integrin $\beta 3 /$ SOCS3/STAT3 signaling pathway
}

\section{Yongyue Gao}

Nanjing University Medical School Affiliated Nanjing Drum Tower Hospital

\section{Zong Zhuang}

Nanjing University Medical School Affiliated Nanjing Drum Tower Hospital

Yue Lu

Nanjing University Medical School Affiliated Nanjing Drum Tower Hospital

\section{Lingyun Wu}

Nanjing University Medical School Affiliated Nanjing Drum Tower Hospital

\section{Guangjie Liu}

Nanjing University Medical School Affiliated Nanjing Drum Tower Hospital

Yan Zhou

Nanjing University Medical School Affiliated Nanjing Drum Tower Hospital

\section{Dingding Zhang}

Nanjing University Medical School Affiliated Nanjing Drum Tower Hospital

\section{Haibin Dai}

Nanjing University Medical School Affiliated Nanjing Drum Tower Hospital

\section{Tao Tao}

Nanjing University Medical School Affiliated Nanjing Drum Tower Hospital

Han Wang

Nanjing University Medical School Affiliated Nanjing Drum Tower Hospital

Wei Li

Nanjing University Medical School Affiliated Nanjing Drum Tower Hospital

Chun-Hua Hang ( $\square$ hang_neurosurgery@163.com )

https://orcid.org/0000-0001-8687-7599

\section{Research}

Keywords: Subarachnoid hemorrhage, MFG-E8 protein, Microglial polarization, Neuro-inflammation, integrin $\beta 3 /$ SOCS3/STAT3 signaling pathway 
Posted Date: March 20th, 2020

DOl: https://doi.org/10.21203/rs.3.rs-18171/v1

License: (c) (i) This work is licensed under a Creative Commons Attribution 4.0 International License. Read Full License 


\section{Abstract}

\section{Background}

Increasing evidence suggests microglial polarization plays an important role in the pathological processes of neuro-inflammation following subarachnoid hemorrhage (SAH). Previous studies indicated that milk fat globule-EGF factor-8 (MFG-E8) has the potential in anti-apoptosis and anti-inflammation in cerebral ischemia. However, the effects of MFG-E8 on microglial polarization have not been evaluated after SAH. Therefore, the aim of this study was to explore the role of MFG-E8 on anti-inflammation, and its potential mechanism on microglial polarization following SAH.

\section{Methods}

We established the SAH model via prechiasmatic cistern Blood injection in mice. Doubleimmunofluorescence staining, Western blotting and quantitative real-time polymerase chain reaction (qPCR) were performed to investigate the expression and cellular distribution of MFG-E8. Two different dosages $(1 \mu \mathrm{g}$ and $5 \mu \mathrm{g}$ ) of recombinant human MFG-E8 (rhMFG-E8) were injected intracerebroventricular (i.c.v.) at $1 \mathrm{~h}$ after $\mathrm{SAH}$. Brain water content, neurological scores, beam-walking score, Fluoro-Jade C (FJC) and terminal deoxynucleotidyl transferase dUTP nick endlabeling staining (TUNEL) were measured at 24 h. Intervention of MFG-E8, integrin $\beta 3$ and phosphorylation of STAT3 were achieved by specific siRNAs $(500 \mathrm{pmol} / 5 \mu \mathrm{l})$ and STAT3 inhibitor Stattic $(5 \mu \mathrm{M})$. The potential signal pathway and microglial polarization were measured by immunofluorescence labeling and Western blotting.

Results

SAH induction increased the levels of inflammation mediators, the proportion of M1 and caused neuronal apoptosis in mice at $24 \mathrm{~h}$. Treatment with rhMFG-E8 $(5 \mu \mathrm{g})$ remarkably decreased brain edema, improved neurological functions, reduced the levels of pro-inflammation factors, and promoted microglia shifted to M2 phenotype. However, knockdown MFG-E8 and integrin $\beta 3$ via siRNA abolished the effects of MFG-E8 on anti-inflammation and M2 phenotype polarization. STAT3 inhibitor Stattic further clarified the role of rhMFG-E8 on microglial polarization through regulating the protein levels of integrin $\beta 3 / S O C S 3 / S T A T 3$ pathway.

\section{Conclusions}

rhMFG-E8 inhibits neuron-inflammation through transformation microglial phenotype towards M2 after SAH, which may be mediated by modulation of the integrin $\beta 3 /$ SOCS3/STAT3 signaling pathway, and highlighting rhMFG-E8 as a potentially therapeutic target for the treatment of SAH patients.

\section{Introduction}

Subarachnoid hemorrhage (SAH), constituting 5 to $10 \%$ of all strokes worldwide, is one of the most catastrophic disease with high morbidity and mortality [1]. Recently, numerous studies indicated that 
early brain injury $(\mathrm{EBI})$ has been considered as the primary cause of poor neurological outcomes $[2,3]$. As one of the major contributors, inflammation injury plays a pivotal role in the pathogenesis of $\mathrm{EBI}$, which is mainly caused by immunological imbalance, including excess release of pro-inflammatory mediators and less production of anti-inflammatory cytokines [4]. Therefore, searching for an effective treatment to regulate immunologic balance and improve the outcome of SAH patient become urgently needed.

Several studies showed that microglia, as the resident innate immune cells of the central nervous system (CNS), is invariably associated with inflammatory responses following SAH $[5,6]$. Microglia could sense the slight imbalances of immune homeostasis, and cytotoxic mediators and endogenous proteins released from damaged nerve cell provoke resting microglia activation [7]. Activated microglia assumed to polarize two phenotypes, including classical M1 (pro-inflammation) and alternative M2 (antiinflammatory) under the different stimulants after SAH [8]. Therefore, the phenotype of M1 are characterized by releasing pro-inflammatory cytokines, including interleukin-1 $\beta$ (IL-1 $\beta$ ), interleukin-6 (IL-6), tumor necrosis factor- $a$ (TNF- $a$ ) and inducible nitric oxide synthase (iNOS), which induces an inflammatory injury. Likewise, M2 produces anti-inflammatory cytokines, such as interleukin-10 (IL-10) and transforming growth factor $\beta$ (TGF- $\beta$ ), to maintain immune balance [9]. Meanwhile, microglia dynamically switch between two phenotypes following activation $[10,11]$. Thus, the anti-inflammatory strategy of targetting microglia phenotypic transformation might provide potential neuro-protection against inflammatory in the treatment of SAH.

Milk fat globule-epidermal growth factor-factor 8 (MFG-E8) is a secreted multifunctional glycoprotein, composed of epidermal growth factor (EGF)-like sequences and discoidin domain-containing protein 1 (SED-1) [12]. This protein is widely distributed in various tissues of mammalian species including humans. Given its role of a bridging molecule between apoptotic cell and macrophages, MFG-E8 facilitate clearance of pro-inflammatory mediators to prevent secondary injury [13]. Recently, its role attracts more and more attentions by researchers in CNS. Normally, MFGE8 is mainly upregulated in microglia during several pathological process, also small amount of it expressed in astrocytes and neurons [14, 15]. MFGE8 participates in regulating immune responses through inhibiting the release of pro-inflammatory mediators to protect against ischemic cerebral injury [13]. Further, MFG-E8 promote microglia reprograming to shift the microglia phenotype toward alternative (M2) activation [16]. Administration of MFG-E8 could alleviate pathological lesion of Alzheimer's disease (AD) via modulating the alteration of M1/M2 polarization [14]. Recently our laboratory have confirmed that recombinant human MFG-E8 (rhMFG-E8) improve neurological function in an animal model of traumatic brain injury (TBI) by inhibiting neuronal apoptosis [17]. These studies indicated that MFG-E8 could provide neuroprotection in CNS. However, whether MFG-E8 modulate the phenotypes and functions of activated microglia after SAH remains to be reported.

In the present work, we aimed to address the effect of MFG-E8 on microglia polarization, as well as its potential mechanism in a SAH model.

\section{Methods}


Adult male C57BL/6J mice weighing 25-28 $\mathrm{g}$ were purchased from the Experimental Animal Center of Drum Tower Hospital. All experimental protocols and procedures for this study were approved by the Institutional Animal Care and Use Committee at Drum tower hospital and conformed to the National Institutes of Health $(\mathrm{NIH})$ Guide for the Care and Use of Laboratory Animals. All mice were acclimated in a 12-h light/dark cycle room, and allowed free access to food and water under conditions of controlled humidity and temperature $\left(24 \pm 0.5^{\circ} \mathrm{C}\right)$.

Models Of SAH And Experimental Design

Experimental SAH models used in this study were performed as previous study [18]. Briefly, mice were placed in a stereotaxic frame after inhalation anesthesia with isoflurane ( $2 \%$ in oxygen gas, $300 \mathrm{ml} / \mathrm{min}$ ). After disinfection, nearly $1.0 \mathrm{~cm}$ midline scalp incision was made and the skull was exposed. A hole with a diameter of $1.0 \mathrm{~mm}$ was drilled through the skull in the midline $4.5 \mathrm{~mm}$ anterior to the bregma. Simultaneously, one mouse was euthanized as a donor for arterial blood by exposed left ventricular cardiac puncture. Next, $50 \mu \mathrm{l}$ arterial blood was injected into the prechiasmatic cistern through the prepared hole, and the needle must kept in this position for at least 2 min to prevent blood backflow or cerebrospinal fluid (CSF) leakage. Sham animals were subjected to the same procedures, but was injected into equal volume of normal saline solution. All animals were allowed to recover for $45 \mathrm{~min}$ after $\mathrm{SAH}$, then returned to the cages and maintained at a temperature of $24.0 \pm 0.5^{\circ} \mathrm{C}$.

All mice were randomly assigned to the following experiments as described (Supplemental Fig. S1).

Experiment design 1 - To determine the expression of MFG-E8 in the cortex of mice after SAH. Mice were randomly assigned to seven groups: Sham group $(n=6)$ and SAH group ( 1 h, 6 h, 12 h, 24 h, 48 h, $72 h)$ $(n=5$ per group, except SAH 24 h group, $n=8) .5$ mice of each group were selected randomly for Western blot analyses and Quantitative Real-Time Polymerase Chain Reaction and the rest mice for doubleimmunofluorescence staining.

Experiment design 2 - To evaluate the effect of rhMFG-E8 on neuroprotection after SAH. Mice were randomly assigned to five group: Sham group, SAH group, SAH + Vehicle group, SAH + rhMFG-E8 $(1 \mu \mathrm{g}$ and $5 \mu \mathrm{g})(\mathrm{n}=16$ each). Assessment method including brain water content and neurological Score $(n=5)$, Western blot analyses $(n=5)$, FJC staining $(n=3)$ and TUNEL-immunofluorescence staining $(n=3)$.

Experiment design 3 - To explore the potential mechanism of rhMFG-E8 on microglial polarization after $\mathrm{SAH}$. Mice were randomly assigned into the following group: Sham group, SAH group, SAH + Vehicle group, SAH + rhMFG-E8, SAH + rhMFG-E8 + Stattic (5 $\mu \mathrm{M})$, SAH + MFG-E8 siRNA, SAH + Scramble siRNA, SAH + rhMFG-E8 + integrin $\beta 3$ siRNA, SAH + rhMFG-E8 + Scramble siRNA. Assessment method including western blot analyses ( $n=5$ per group) and double immunofluorescence staining ( $n=3$ per group).

Intracerebroventricular Administration 
Intracerebroventricular (i.c.v) drug administration was performed as previously described [18]. Briefly, mice were placed in a stereotaxic frame after inhalation anesthesia with isoflurane ( $2 \%$ in oxygen gas,

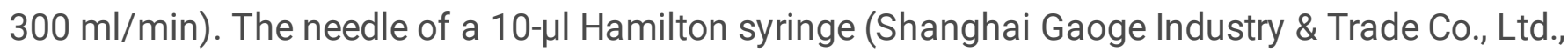
Shanghai, China) was inserted into the left lateral ventricle through a burr hole using the following coordinates: $1.0 \mathrm{~mm}$ posterior and $1.5 \mathrm{~mm}$ lateral to the bregma, and $3.2 \mathrm{~mm}$ below the dural layer. rhMFG-E8 was purchased from R\&D Systems, Inc. (McKinley, NE, USA) and injected at $1 \mathrm{~h}$ after SAH induction, doses of rhMFG-E8 were determined according to previous study [17, 19]. Stattic (Abcam, USA, $5 \mu \mathrm{M}$ ) was dissolved in sterile saline solution containing $2 \%$ dimethyl sulfoxide (DMSO) and injected at $1 \mathrm{~h}$ before SAH induction. According to the detection of siRNA effect and previous study [17, 19], MFG-E8 siRNA, integrin $\beta 3$ siRNA and scramble siRNA ( $500 \mathrm{pmol} / 5 \mu \mathrm{l}$, Santa Cruz Biotechnology) were injected into the left lateral ventricles at a rate of $0.5 \mu \mathrm{l} / \mathrm{min}$ with a $10-\mu \mathrm{l}$ Hamilton syringe at $2 \mathrm{~d}$ before SAH induction.

Quantitative Real-time Polymerase Chain Reaction

Quantitative real-time polymerase chain reaction (qPCR) was performed and analyzed as previously described [20]. Total RNA from brain tissues was extracted using TRIzol Reagents (Invitrogen Life Technologies, USA). RNA quality was insured by gel visualization and spectrophotometric analysis $\left(\mathrm{OD}_{260 / 280}\right)$. After reverse transcription, quantitative analysis of the MFG-E8, Integrin $\beta 3$, IL-1 $\beta$, IL-6, TNF-a and IL-10 mRNA expression were performed with the real-time PCR method and the primers were synthesized by ShineGene Biotechnology (Shanghai, China) (Additional file Table 1). Test cDNA results were normalized to $\beta$-actin. All samples were analyzed in triplicate.

Table 1

Polymerase chain reaction (PCR) primer sequences.

\begin{tabular}{|c|c|c|}
\hline Target gene & Forward ( $\left(5^{\prime}\right.$ to $\left.3^{\prime}\right)$ & Reverse ( $5^{\prime}$ to $\left.3^{\prime}\right)$ \\
\hline mfg-e8 & CGGGCCAAGACAATGACATC & TCTCTCAGTCTCATTGCACACAAG \\
\hline gapdh & AAGAAGGTGGTGAAGCAGGC & TCCACCACССTGTTGCTGTA \\
\hline il-1 $\beta$ & AAGCCTCGTGCTGTCGGACC & TGAGGCCCAAGGCCACAGG \\
\hline il-6 & GAGACTTCCATCCAGTTGCCT & TGGGAGTGGTATCCTCTGTGA \\
\hline tnf-a & GTAGCCCACGTCGTAGCAAA & СССТTСTCCAGCTGGAAGAC \\
\hline il-10 & GGTTGCCAAGCCTTATCG A & АСCTGCTCCACTGCCTTGCT \\
\hline$\beta$-actin & CGTGAAAAGATGACCCAGATCA & CACAGCCTGGATGGCTACGTA \\
\hline
\end{tabular}


Table 2

Neurological evaluation (Modified Garcia scoring)

\begin{tabular}{|lllll|}
\hline Test & Score & & & \\
\cline { 2 - 4 } & $\mathbf{0}$ & $\mathbf{1}$ & $\mathbf{2}$ & Move but does not \\
\hline $\begin{array}{l}\text { Spontaneous } \\
\text { Activity } \\
\text { (in cage for } \\
5 \text { min) }\end{array}$ & $\begin{array}{l}\text { No } \\
\text { movement }\end{array}$ & $\begin{array}{l}\text { Barely } \\
\text { moves } \\
\text { position }\end{array}$ & $\begin{array}{l}\text { Move and } \\
\text { approach at least three } \\
\text { sidef cage }\end{array}$ & $\begin{array}{l}\text { approaches at least } \\
\text { three sides of cage }\end{array}$ \\
\hline $\begin{array}{l}\text { Spontaneous } \\
\text { movements of all } \\
\text { limbs }\end{array}$ & $\begin{array}{l}\text { No } \\
\text { movement }\end{array}$ & $\begin{array}{l}\text { Slight } \\
\text { movement } \\
\text { of limbs }\end{array}$ & $\begin{array}{l}\text { Moves all limbs but } \\
\text { slowly }\end{array}$ & $\begin{array}{l}\text { Move all limbs same } \\
\text { as pre-SAH }\end{array}$ \\
$\begin{array}{l}\text { Movements of } \\
\text { forelimbs } \\
\text { (outstretching } \\
\text { while held by tail) }\end{array}$ & No & $\begin{array}{l}\text { Slight } \\
\text { outreaching }\end{array}$ & $\begin{array}{l}\text { Outreach is limited and } \\
\text { less than pre-SAH }\end{array}$ & $\begin{array}{l}\text { Outreach same as } \\
\text { pre-SAH }\end{array}$ \\
$\begin{array}{l}\text { Climbing wall of } \\
\text { wire cage }\end{array}$ & $\begin{array}{l}\text { Fails to } \\
\text { climb }\end{array}$ & Climbs weakly & Normal climbing \\
\hline $\begin{array}{l}\text { Reaction to touch } \\
\text { on both side of } \\
\text { trunk }\end{array}$ & $\begin{array}{l}\text { No } \\
\text { response }\end{array}$ & Weak response & Normal response \\
\hline $\begin{array}{l}\text { Response to } \\
\text { vibrissae touch }\end{array}$ & $\begin{array}{l}\text { No } \\
\text { response }\end{array}$ & Weak response & Normal response \\
\hline
\end{tabular}

Immunofluorescence Staining And TUNEL Staining

Immunofluorescence Staining was performed as previously described [18, 21]. Briefly, mice were deeply euthanized and perfused with $4 \%$ paraformaldehyde in $0.1 \mathrm{mM}$ phosphate-buffered saline (PBS, PH7.4). Brain samples were immersed in $30 \%$ sucrose until sinking to the bottom. 8 um-thick slices were cut with a cryostat. The slides of each coronal sections were incubated in blocking buffer for $2 \mathrm{~h}$, then washed with PBS three times for $10 \mathrm{~min}$. Next, the slides were incubated with anti-MFG-E8 (1:200), anti-CD86 antibody (1:100), anti-CD206 antibody (1:100), and anti-p-STAT3 (1:200) respectively, in a dark place overnight at $4{ }^{\circ} \mathrm{C}$. Afterwards, the slides were washed three times with PBS and incubated with another antibody, namely anti-NeuN (1:100), anti-GFAP (1:100), anti-Iba-1 (1:50), under similar conditions. The following day, the slides were thoroughly washed with PBST and incubated with the corresponding secondary antibodies for $1 \mathrm{~h}$ at the room temperature. For TUNEL staining, the sections were incubated with the TUNEL reagent for $1 \mathrm{~h}$ at $37^{\circ} \mathrm{C}$. Then wash with PBS, the slides were stained with DAPI for 15 min to show the location of nucleus. Coverslips were applied with mounting media. The fluorescentlystained cells were imaged on an Olympus IX71 inverted microscope system and analyzed using the Image-Pro Plus 6.0 software (Media Cybernetics, Silver Spring, MD, USA).

Western Blot Analysis 
For Western blot analysis, the total protein concentration of the lysate was determined by the Bradford method using Bradford Protein Assay Kit (Beyotime Biotechnology, Shanghai, China). Equal amounts of proteins were resolved on a 10\%-12\% sodium dodecyl sulfate-polyacrylamide gel electrophoresis (SDSPAGE) gel and transferred onto polyvinylidene fluoride (PVDF) membrane (Immobilon-P, Millipore Billerica MA, USA). Then, blocked with $5 \%$ non-fat dry milk in TBST (Tris-buffered saline with $0.05 \%$ Tween 20 ) for $2 \mathrm{~h}$ at room temperature, and then incubated overnight at $4{ }^{\circ} \mathrm{C}$, separately with the appropriate primary antibodies against the specific proteins, MFG-E8, IL-1 $\beta$, IL-6, TNF-a, IL-10, Integrin $\beta 3$ (Santa Cruz Biotechnology, USA, 1:200), CD86, CD206 (Abcam, Cambridge, UK, 1:200), STAT3, p-STAT3 at Try 705, and SOCS3 (Cell Signaling Technology, United states, 1:1000), $\beta$-actin (Bioworld Technology, United states, 1:5000), and GAPDH (Cell Signaling Technology, United states, 1:5000) in a blocking buffer. Afterwards, the membrane was washed three times with TBST for $15 \mathrm{~min}$, and then incubated with the secondary antibodies, namely HRP conjugated secondary antibodies (goat; Bioworld Technology, United states, 1:5000) or HRP conjugated secondary antibodies (horse; Cell Signaling Technology, United states, 1:1000) for $2 \mathrm{~h}$ at room temperature. Finally, following a 20-min wash with TBST, the protein bands were visualized via enhanced chemiluminescence (ECL) (Millipore, Billerica, MA, USA) and exposure to X-ray film. The Western blot results were analyzed using Un-Scan-It 6.1 software (Silk Scientific Inc., Orem, UT, USA).

Fluoro-jade C (FJC) Staining

FJC staining (Merckmillipore, Germany) was performed according to the operation instructions and to detect degenerating neurons. Briefly, frozen sections were prepared, fixed, and immersed in a basic alcohol solution consisting of $1 \%$ sodium hydroxide in $80 \%$ ethanol for $5 \mathrm{~min}$, then rinsed for $2 \mathrm{~min}$ each in $70 \%$ ethanol and distilled water and then incubated in $0.06 \%$ potassium permanganate solution for $10 \mathrm{~min}$. Following a 1-2 min water rinse, the slides were transferred for $10 \mathrm{~min}$ to a $0.0001 \%$ solution of FJC dissolved in $0.1 \%$ acetic acid vehicle and then rinsed through three changes of distilled water for 1 min per change. The slides were air-dried, cover slips were applied, and the sections were visualized on an Image $\mathrm{J}$ software (Image $\mathrm{J} 1.4, \mathrm{NIH}$, USA). Two observer blinded to the experimental group counted the FJC-positive cells in six sections per brain (at $20 \times$ magnification) through the injury's epicenter. The data were presented by the average number of FJC-positive neurons in the fields.

\section{Brain Water Content}

Brain water content was measured as previously study [22, 23]. Brains were quickly removed at $24 \mathrm{~h}$ after $\mathrm{SAH}$. The brainstem was discarded, while the tissue of left hemisphere cortex and right cortex were harvested, and weighted the wet weight of each cortical tissue, then dried for $72 \mathrm{~h}$ at $80^{\circ} \mathrm{C}$ and the dry weight determined. The percentage of brain water content was calculated as the following formula $=$ [(wet weight - dry weight)/wet weight $] \times 100 \%$.

Neurologic Evaluation 
The neurological deficits were evaluated as previously described modified Garcia scoring (Additional file Table. 2) and beam-walking tests at $24 \mathrm{~h}$ after SAH [24-26]. Briefly, modified Garcia scoring (maximum score $=18$ ) included six subtests scored from 0 to 3 or 1 to 3 : spontaneous activity, spontaneous movement of four limbs, forelimbs outstretching, climbing ability, body proprioception, and the response to vibrissae stimulation. Beam-waling texts were performed and including seven-point rating scale. All the tests were evaluated by two independent observer who was blind to the treatment conditions. Higher scores represented better neurological function.

Statistical Analysis

The SPSS 17.0 software package was used for the statistical analysis. All data are expressed as the mean \pm Standard Deviation (SD). Comparisons between two groups were performed using Student's $t$ test and multiple comparisons were performed using a one-way ANOVA followed by Tukey's test. A $p$ value < 0.05 was regarded as statistically significant.

\section{Results}

General observations and mortality rate

Out of the 217 surgeries in mice that were performed. The mortality rate of SAH group on rhMFG-E8, siRNA and Stattic treatment did not differ significantly from the SAH group and SAH + Vehicle group. No rats died in Sham group 0\% (0/38), and the overall mortality rate of SAH in mice was $17.97 \%$ (Additional file Table 3). There was no statistical difference in body weight and body temperature in any of the experimental groups (data not shown). 
Table 3

Mortality rate following SAH in mice

\begin{tabular}{|c|c|c|}
\hline Groups & Death number (n) & Mortality Rate (\%) \\
\hline \multicolumn{3}{|l|}{ Experiment design 1} \\
\hline Sham & 0 & $0(0 / 6)$ \\
\hline SAH ( 1 h, 6 h, 12 h, 24 h, 48 h, 72 h) & 5 & $15.15 \%(5 / 33)$ \\
\hline \multicolumn{3}{|l|}{ Experiment design 2} \\
\hline Sham & 0 & $0(0 / 16)$ \\
\hline $\mathrm{SAH}$ & 2 & $11.11 \%(2 / 18)$ \\
\hline SAH + Vehicle & 3 & $15.79 \%(3 / 19)$ \\
\hline SAH + rhMFG-E8 $(1 \mu \mathrm{g})$ & 3 & $15.79 \%(3 / 19)$ \\
\hline SAH + rhMFG-E8 $(5 \mu \mathrm{g})$ & 2 & $11.11 \%(2 / 18)$ \\
\hline \multicolumn{3}{|l|}{ Experiment design 3} \\
\hline \multicolumn{3}{|l|}{ Groups ${ }^{1 *}$} \\
\hline Sham & 0 & $0(0 / 8)$ \\
\hline SAH & 1 & $11.11 \%(1 / 9)$ \\
\hline SAH + Vehicle & 2 & $20 \%(2 / 10)$ \\
\hline SAH + rhMFG-E8 $(5 \mu \mathrm{g})$ & 2 & $20 \%(2 / 10)$ \\
\hline \multicolumn{3}{|l|}{ Groups $^{2 *}$} \\
\hline SAH + Vehicle & 1 & $11.11 \%(1 / 9)$ \\
\hline $\mathrm{SAH}+$ rhMFG-E8 $(5 \mu \mathrm{g})$ & 2 & $20 \%(2 / 10)$ \\
\hline SAH + MFG-E8 siRNA & 2 & $20 \%(2 / 10)$ \\
\hline SAH + Scramble siRNA & 3 & $27.27 \%(3 / 11)$ \\
\hline \multicolumn{3}{|l|}{ Groups ${ }^{3 *}$} \\
\hline Sham & 0 & $0(0 / 8)$ \\
\hline SAH + Vehicle & 2 & $20 \%(2 / 10)$ \\
\hline SAH + rhMFG-E8 $(5 \mu \mathrm{g})$ & 3 & $27.27 \%(3 / 11)$ \\
\hline SAH + rhMFG-E8 $(5 \mu \mathrm{g})+$ Stattic $(5 \mu \mathrm{M})$ & 1 & $11.11 \%(1 / 9)$ \\
\hline SAH + rhMFG-E8 $(5 \mu \mathrm{g})+$ integrin $\beta 3$ siRNA & 2 & $20 \%(2 / 10)$ \\
\hline
\end{tabular}




\begin{tabular}{|lll|}
\hline Groups & Death number $(\mathbf{n})$ & Mortality Rate (\%) \\
\hline SAH + rhMFG-E8 $(5 \mu \mathrm{g})+$ Scramble siRNA & 3 & $27.27 \%(3 / 11)$ \\
\hline Total & & \\
\hline Sham & 0 & $0(0 / 38)$ \\
\hline SAH & 39 & $17.97 \%(39 / 217)$ \\
\hline
\end{tabular}

The endogenous MFG-E8 increased and mainly expressed in microglia and neuron at $24 \mathrm{~h}$ after SAH

Firstly, we examined the endogenous expression of MFG-E8 on left temporal cortex via quantitative realtime PCR and Western blot in the model of SAH. There were significant elevations of MFG-E8 on protein and mRNA levels at the $6 \mathrm{~h}$ time point compared with the sham group, which reached its peaks at $24 \mathrm{~h}$ and $12 \mathrm{~h}$, respectively (Fig. 1A, B). Then, we confirmed the cellular distribution of MFG-E8 by doubleimmunofluorescence staining at $24 \mathrm{~h}$ following $\mathrm{SAH}$, the time relying on the result of western blot. As shown in Fig. 1C, MFG-E8 mainly expressed in the microglia and neurons, especially in microglia after SAH compared with sham group, and without observed in astrocytes.

Treatment with rhMFG-E8 alleviated neuronal damage, brain edema and improved neurobehavioral outcome after SAH

To investigate whether MFG-E8 had effect of neuroprotection after SAH, brain edema and neurological score were examined, which revealed that SAH induction aggravated brain water content and neurological impairments compared with sham group at $24 \mathrm{~h}$. While administration two different dosages of rhMFG-E8 $(1 \mu \mathrm{g}$ and $5 \mu \mathrm{g})$ via intracerebroventricular (i.c.v.), brain water content was remarkably alleviated and neurological deficits was dramatically recovered compared with $\mathrm{SAH}+$ Vehicle group. Moreover, high dosage of rhMFG-E8 performed more effective on neuroprotection (Fig. 2A-D).

FJC and TUNEL staining were detected to reflect the neurodegeneration and neuronal apoptosis at $24 \mathrm{~h}$ following SAH. As shown in Fig. 2E, large number of FJC-positive cells were observed in SAH group and $\mathrm{SAH}+$ Vehicle group compared with sham group. Treatment with rhMFG-E8 significantly reduced the proportion of FJC-positive cells, while the rhMFG-E8 group with low dosage had no statistical difference relative to the SAH + Vehicle group. TUNEL staining showed a similar pattern. SAH induction remarkably increased the amount of TUNEL-positive neurons in SAH group and SAH + Vehicle group compared with sham group, and administration of rhMFG-E8 significantly decreased the number of TUNEL-positive neurons (Fig. 3). No statistical differences of FJC and TUNEL staining between SAH group and SAH + Vehicle group, respectively.

rhMFG-E8 attenuated inflammation and promoted microglia phenotype shifted to M2 at $24 \mathrm{~h}$ after SAH To investigate the potential role of rhMFG-E8 on neuro-inflammation, we first examined the protein and mRNA levels of interleukin 1区 (IL-1区), interleukin 6 (IL-6), tumor necrosis factor a (TNF-a) and interleukin 
10 (IL-10) on the temporal cortex. The results showed that the expression of IL-1区, IL-6, TNF-a and IL-10 on the protein levels were elevated after SAH induction at $24 \mathrm{~h}$. However, treated with rhMFG-E8 significantly decreased the expression of pro-inflammatory cytokines (IL-1区, IL-6 and TNF-a) and promoted release of anti-inflammatory cytokines IL-10 (Fig. 4A-E). Meanwhile, IL-6 and TNF-a protein level had no significantly changes in the group with low dosage rhMFG-E8 $(1 \mu \mathrm{g})$ compared with the SAH + Vehicle group. Consistent with the results above. mRNA levels of IL-1区, IL-6, TNF-a and IL-10 were remarkably elevated at 24 after SAH. Only high dosage of rhMFG-E8 (5 $\mu \mathrm{g})$ treatment markedly reversed the expressions of these cytokines relative to the SAH + Vehicle group (Fig. 4F-I). Thus, we selected the high dosage for the following studies.

To evaluate whether MFG-E8 is relevant to modulate microglial polarization, the protein levels of M1associated marker (CD86) and M2-associated marker (CD206) were measured by Western blots and immunofluorescent staining. The results showed that the proportions of $M 1$ and $M 2$ phenotypes, revealed by $\mathrm{Iba}-1^{+} / \mathrm{CD} 86^{+}$and $\mathrm{Iba}-1^{+} / \mathrm{CD} 206^{+}$staining respectively, were upregulated after $\mathrm{SAH}$ at $24 \mathrm{~h}$ compared with sham group (Fig. 5A, D). While administration of rhMFG-E8 significantly decreased the number of $\mathrm{Iba}-1^{+} / \mathrm{CD} 86^{+}$cell, and upregulated the ratio of $\mathrm{Iba}-1^{+} / \mathrm{CD} 206^{+}$cell compared with the SAH + Vehicle group (Fig. 5B, E). Similarly, Western blot results showed that SAH induction increased the protein levels of CD86 and CD206 in SAH group and SAH + Vehicle group compared to sham group, while treated with rhMFG-E8 remarkably reduced the expression of CD86, meanwhile further increased the protein level of CD206 (Fig. 5C, F). Considering with these results above, we speculated that the property of MFG-E8 on anti-inflammation might involves microglial polarization, namely, declined the proportion of proinflammation M1 phenotype and amplified anti-inflammation M2 polarization.

Knockdown MFG-E8 aggravated neuro-inflammation injury and induced M1 microglia activation

To confirm the effects of MFG-E8 on microglia phenotypic conversion process, MFG-E8 siRNA at the concentration of $500 \mathrm{pmol} / 5 \mu \mathrm{l}$ were used injecting via i.c.v. $48 \mathrm{~h}$ before $\mathrm{SAH}$. As shown in Fig. 6A, the number of $\mathrm{Iba}^{-} \mathrm{1}^{+} / \mathrm{CD} 86^{+}$was significant increased after treatment with MFG-E8 siRNA at SAH group compared with SAH + rhMFG-E8 group. Meanwhile, Western blot results showed that MFG-E8 siRNA upregulated the expression of $\mathrm{M} 1$ markers (CD86, IL-6) relative to the SAH + rhMFG-E8 group (Fig. 6C-E). While the proportion of Iba- $1^{+} / \mathrm{CD} 206^{+}$was remarkably reduced (Fig. 6F, G) and the protein levels of M2 markers (CD206, IL-10) greatly attenuated (Fig. 6H-J), when compared with the SAH + rhMFG-E8 group. As expected, there was no statistical difference between the SAH + Vehicle group and SAH + Scramble siRNA group. Taken together, we concluded that rhMFG-E8 was involved in the regulation of microglial polarization process after $\mathrm{SAH}$.

Knockdown integrin $\beta 3$ and STAT3 inhibition exaggerated M1 microglia polarization and abolished the property of MFG-E8 on anti-inflammation after SAH

STAT3, as an important transcription factor, SOCS3/STAT3 signaling pathway was involved in neuroinflammation and microglial polarization. Since phosphorylation STAT3 was mainly expressed in 
microglia, so we detected its phosphorylation level via double-immunofluorescence staining in microglia. The result showed that SAH induction significantly increased fluorescence intensity of p-STAT3 at SAH + Vehicle group, compared with sham group. Additionally, some of p-STAT3 translocated from cytoplasm to nucleus in $\mathrm{Iba}-1^{+}$microglia at $\mathrm{SAH}+$ Vehicle group, while, it were significantly decreased underwent rhMFG-E8 and STAT3 inhibitor Stattic $(5 \mu \mathrm{M})$ treatment. Subsequently, intervention with integrin $₫ 3$ siRNA enhanced the fluorescence intensity of p-STAT3 and promoted the protein transfer into nucleus of microglia (Fig. 7). This result suggested that MFG-E8 and integrin $₫ 3$ receptor might be involved in the phosphorylation of STAT3 in microglia after SAH.

Therefore, for further exploring the regulatory mechanism of MFG-E8 on microglial polarization, SOCS3/STAT3 axis were examined via Western blots. Treated with rhMFG-E8 significantly increased the expression of integrin $\varangle 3$, SOCS3, IL 10 and CD206 after SAH, meanwhile, decreased the levels of p-STAT3, IL-6 and CD86 when compared with the SAH + Vehicle group (Fig. 8A, E). Subsequently, we used integrin $₫ 3$ siRNA to examine the association between MFG-E8 and p-STAT3. Western blot results showed that integrin $₫ 3$ siRNA treatment remarkably reduced the expression of integrin $₫ 3$ compared with $\mathrm{SAH}+$ Vehicle group and SAH + rhMFG-E8 group (Fig. 8B). However, integrin $₫ 3$ siRNA reversed the role of rhMFG-E8 on SOCS3/STAT3 axis, M1 markers (IL-6, CD86) (Fig. 8F, H) and M2 markers (IL-10, CD206) (Fig. 8G, I). In other words, integrin $₫ 3$ siRNA abolished the effect of rhMFG-E8 on M2 polarization and activation of SOCS3/STAT3 pathway.

Correspondently, intervention of Stattic resulted in remarkably reduction of p-STAT3 (Fig. 8D), meanwhile the expression of M2 markers (IL10, CD206) elevated dramatically and the levels of M1 markers (IL-6, CD86) decreased in comparison with the SAH + rhMFG-E8 group. However, Stattic had no effect on the protein levels of integrin $₫ 3$ and SOCS3 compared with the SAH + rhMFG-E8 group (Fig. 8B, C). These results suggested that rhMFG-E8 played an important role in M2 polarization, which might be through regulating integrin $₫ 3 /$ SOCS3/STAT3 signal pathway.

\section{Discussion}

Previous study reported that neuro-inflammatory response was the main mechanism pathological damage of early brain damage following $\mathrm{SAH}$, and regulating inflammatory response can effectively improve the prognosis of SAH patients [27]. In this study, we revealed the neuroprotective effect of rhMFG-E8, mainly through targeting the immunomodulatory functions in animal SAH models. The results showed that the expression of MFG-E8 was time-dependent increased at an early stage of SAH, and which was almost located in microglia and neurons cells. Exogenous rhMFG-E8 effectively attenuated brain edema and improved neurological deficits. rhMFG-E8 up-regulated the expression of M2 microglia function-related proteins and took a significant role in $\mathrm{M} 2$ phenotypic shift. Additionally, our results suggested that integrin $\beta 3 /$ SOCS3/STAT3 signaling pathway might be involved in the neuroprotective effects of MFG-E8. 
MFG-E8 is a secretory glycoprotein and plays pleiotropic, nonredundant roles in diverse physiological functions [28]. Recently, increasing evidence indicated that MFG-E8 provided neuroprotective effects in several models of CNS diseases, most of which focused on neuron apoptosis [12], anti-oxidative stress [19], and particularly the anti-inflammatory aspect [29]. As reported, in a rat model of postischemic cerebral injury, MFG-E8 interfered the release of inflammasome-mediated proinflammatory mediators IL$1 \beta$ and TNF-a, and reduced infarct volume [13]. In our previous study, we reported that the expressions of pro-apoptosis related proteins and the proportion of apoptotic neurons were attenuated in TBI model after treatment with rhMFG-E8, accompanied by improved neurological performance. This protection was mediated through activation of integrin $\beta 3 / F A K / P I 3 K / A K T$ signal pathways [17]. All this studies indicated that MFG-E8 had potential therapeutic benefits in the brain. In this study, we found that the protein of MFG-E8 was positively co-expressed not only in microglia and but also in neuron following SAH, especially in $\mathrm{Iba} 1^{+}$microglia. We also found that supplementation of rhMFG-E8 induced a significant reduction of brain edema and preserved neurological function. Correspondingly, down-regulation the expression of MFG-E8 via siRNA aggravated outcomes after SAH. Our results clearly indicated that there has a closely relationship between the level of MFG-E8 and severity of brain damage, and consistent with the other studies to reflect the neuroprotection effects of MFG-E8.

Accumulating evidence indicated that the neuroinflammation increasingly recognized as a key player to the pathophysiology of various brain injury diseases, including ischemia [30], neurodegeneration [31], SAH [32] or intracerebral hemorrhage (ICH) [9]. Microglia as the resident immunocyte of CNS activated and shifted towards proinflammatory-status phenotypes to exacerbate brain damage [33]. Although microglia activated into the controversial dichotomy between $\mathrm{M} 1$ and $\mathrm{M} 2$ phenotypes, the process termed polarization, this simply assortment will useful for clarifying the character of microglia in several brain diseases. Meanwhile, as the main source of proinflammation mediators, apoptotic cells release amounts of cell debris and damage-associated molecular patterns (DAMPs) to induce inflammation cascades and microglia activation [34]. MFG-E8 as a bridging protein play an important role in mediating microglia phagocytosis, which connect between the integrin receptors expressed by microglia and phosphatidylserine (PS) exposed on apoptotic cells [35]. Moreover, MFG-E8 regulated inflammatory responses through activation of microglia and phenotypic transformation when its bind to integrin molecule [14]. In our study, we found that microglia activated in SAH group and shifted to M1, produce more proinflammation cytokines including IL-1 $\beta, T N F-a$ to aggravate neuron cells injury. However, treatment with rhMFG-E8 significantly reversed the expressions of CD86 and mannose receptors (CD206), which are markers of M1 and M2, respectively. Meanwhile, our results showed that intervening the level of integrin $\beta 3$ receptor on microglia abrogated the effects of rhMFG-E8 on anti-inflammation. Based on these findings, we speculate that the role of rhMFG-E8 on balance of microglia polarization might through interaction with integrin $\beta 3$ receptor.

How does rhMG-E8 mediate the microglia shifted to $\mathrm{M} 2$ after $\mathrm{SAH}$ ? It was reported that phosphorylation of STAT3 was involved in microglia-induced inflammation responses and microglia polarization in various diseases $[8,36]$. Then we speculated that whether rhMFG-E8 might through bind to integrin $\beta 3$ 
receptor to modulate the expression of STAT3. However, it's still controversial whether activated STAT3 in microglia functions as an inflammation or neuroprotection in pathological condition [37, 38]. Previous study showed that down-regulation the phosphorylation of STAT3 via siRNA in ischemic cerebral injury provided neuroprotection [11]. Similarly, our study showed that the expression of phosphorylation of STAT3 at tyrosine 705 locus increased, accompanied by increasing inflammation responses following SAH. Furthermore, rhMFG-E8 was found to down-regulate the phosphorylation of STAT3 and provide antiinflammatory effect. Whereas intervening integrin $\beta 3$ receptor via siRNA reversed the effect of rhMFG-E8 on phosphorylation of STAT3 and M2 polarization after SAH. Depending on these results, we discovered that rhMFG-E8 might interact with integrin $\beta 3$ receptor to effectively decreased the phosphorylation of STAT3 following SAH. Therefore, this arouses our interest to elucidate how MFG-E8/integrin $\beta 3$ receptor signaling to modulate the phosphorylation of STAT3. Integrin receptors, as a complex component could receive the extra-cellular signaling and cross talk with other intra-cellular pathways [36]. As we all known, once its ligand MFG-E8 combined with integrin $\beta 3$ receptor, amounts of intra-cellular molecules such as focal adhesion kinase (FAK), Src and caveolin would generate and transduce into different signaling [39]. Our previous study also showed that MFG-E8 could activate the AKT signaling through integrin $\beta 3$ mediated phosphorylation of FAK for promoting neurons survival [17]. Meanwhile, Behera et al found that the ligand of integrin $\beta 3$, osteopontin (OPN) enhanced tumor progression through activation of integrin $\beta 3$ mediated STAT3 in breast cancer [40]. From these evidences, we anticipated that MFG-E8 might regulate the phosphorylation of STAT3 via integrin $\beta 3$ receptor. Our study also discovered that increased rhMFG-E8 and integrin $\beta 3$ expression correlate with attenuated inflammation, accompanied by increased the expression of SOCS3. Recent study showed that over-activation of STAT3 might due to the absence of SOCS3 on normal feedback mechanism following pathological stress. Our results were consistent with previous study to underline the possible mechanisms of rhMFG-E8 on anti-inflammation and M2 polarization [14]. In short, we speculated rhMFG-E8 might induce SOCS3 activation through interacting with integrin $\beta 3$ and interfering with phosphorylation of STAT3 to reduce inflammation after SAH.

As far as we know, we demonstrated for the first time that MFG-E8 confers anti-inflammation and microglia polarization partly depending on the integrin $\beta 3 /$ SOCS3/STAT3 signaling pathway following $\mathrm{SAH}$. Additionally, our study mainly focused on the immunomodulation of rhMFG-E8 during the EBI of SAH. However, the long-term effect of rhMFG-E8 on microglia polarization have not been investigation, and should be studied in the future. Moreover, all of the intervention agents we used in our study were siRNAs and Stattic (STAT3 inhibitor). Therefore, the knockout mice need to be used in the further study to keep the data more persuasive.

\section{Conclusions}

In conclusion, we demonstrate that MFG-E8 provides neuroprotection via modulation of inflammation after SAH. Treatment with rhMFG-E8 alleviate microglia inflammation response, which relate to mediating M2 microglia shift, and might involve the mechanism of integrin $33 /$ SOCS3/STAT3 signaling pathway. Therefore, MFG-E8 may become a promising candidate to suppress microglia mediated neuroinflammation and improve the clinical outcomes of SAH patients. 


\section{Abbreviations}

rhMFG-E8: recombinant human milk fat globule-EGF factor-8®SAH: subarachnoid hemorrhage; EBI: early brain injury; CNS: central nervous system; CSF: Cerebrospinal fluids; DAMP: Damage-associated molecular pattern; IL-1 $\beta$ : Interleukin 1 $\beta$; IL-6: Interleukin 6; TNF-a: Tumor necrosis factor-a; IL-10: Interleukin 10; iNOS: inducible nitric oxide synthase; TFG- $\beta$ : transforming growth factor; EGF: epidermal growth factor; FAK: focal adhesion kinase

\section{Declarations}

\section{Ethics approval and consent to participate}

All experimental protocols and procedures for this study were approved by the Institutional Animal Care and Use Committee at Drum tower hospital and were conducted in accordance with the National Institutes of Health $(\mathrm{NIH})$ Guide for the Care and Use of Laboratory Animals.

\section{Consent for publication}

Not applicable

\section{Availability of data and material}

Not applicable.

\section{Competing interests}

The authors declare no conflict of interest.

\section{Funding}

This work was supported by the National Natural Science Foundation of China (No. 81771291 for C.H. Hang, NO. 81870922 for W. Li), Key Project supported by Medical Science and technology development Foundation, Nanjing Department of Health (NO. JQX18001 for W. Li), Fundamental Research Funds for the Central Universities (NO. 021414380361 for W. Li)

\section{Authors' contributions}

YYG conceived and designed the project, biochemical analysis and wrote the manuscript. ZZ performed the SAH model and analyzed the data of the animal studies. YL performed the real-time polymerase chain reaction assay. LYW, GJL, and YZ performed the immunofluorescence staining. DDZ, HBD, and TT provided the data of Western blotting. HW provided the experimental technical support. WL and C-HH contributed to the design and provided critical revisions. All authors checked and approved the final manuscript. 
Acknowledgements

Not applicable.

\section{References}

1. van Gijn J, Kerr RS, Rinkel GJE: Subarachnoid haemorrhage. The Lancet 2007, 369:306-318.

2. Ayling OG, Ibrahim GM, Alotaibi NM, Gooderham PA, Macdonald RL: Dissociation of Early and Delayed Cerebral Infarction After Aneurysmal Subarachnoid Hemorrhage. Stroke 2016, 47:29452951.

3. Zhang X, Wu Q, Lu Y, Wan J, Dai H, Zhou X, Lv S, Chen X, Zhang X, Hang C, Wang J: Cerebroprotection by salvianolic acid $B$ after experimental subarachnoid hemorrhage occurs via Nrf2- and SIRT1-dependent pathways. Free Radical Biology and Medicine 2018, 124:504-516.

4. Zhang X, Lu Y, Wu Q, Dai H, Li W, Lv S, Zhou X, Zhang X, Hang C, Wang J: Astaxanthin mitigates subarachnoid hemorrhage injury primarily by increasing sirtuin 1 and inhibiting the Toll-like receptor 4 signaling pathway. FASEB J 2019, 33:722-737.

5. Rappert A, Bechmann I, Pivneva T, Mahlo J, Biber K, Nolte C, Kovac AD, Gerard C, Boddeke HW, Nitsch $\mathrm{R}$, Kettenmann $\mathrm{H}$ : CXCR3-dependent microglial recruitment is essential for dendrite loss after brain lesion. J Neurosci 2004, 24:8500-8509.

6. Li Q, Barres BA: Microglia and macrophages in brain homeostasis and disease. Nat Rev Immunol 2018, 18:225-242.

7. Salter MW, Stevens B: Microglia emerge as central players in brain disease. Nat Med 2017, 23:10181027.

8. Li R, Liu W, Yin J, Chen Y, Guo S, Fan H, Li X, Zhang X, He X, Duan C: TSG-6 attenuates inflammationinduced brain injury via modulation of microglial polarization in SAH rats through the SOCS3/STAT3 pathway. J Neuroinflammation 2018, 15:231.

9. Lan X, Han X, Li Q, Yang QW, Wang J: Modulators of microglial activation and polarization after intracerebral haemorrhage. Nat Rev Neurol 2017, 13:420-433.

10. Butovsky 0 , Weiner HL: Microglial signatures and their role in health and disease. Nat Rev Neurosci 2018, 19:622-635.

11. Meng H-L, Li X-X, Chen Y-T, Yu L-J, Zhang H, Lao J-M, Zhang X, Xu Y: Neuronal Soluble Fas Ligand Drives M1-Microglia Polarization after Cerebral Ischemia. CNS Neuroscience \& Therapeutics 2016, 22:771-781.

12. Cheyuo C, Jacob A, Wu R, Zhou M, Qi L, Dong W, Ji Y, Chaung WW, Wang H, Nicastro J, et al: Recombinant human MFG-E8 attenuates cerebral ischemic injury: Its role in anti-inflammation and anti-apoptosis. Neuropharmacology 2012, 62:890-900.

13. Deroide N, Li X, Lerouet D, Van Vre E, Baker L, Harrison J, Poittevin M, Masters L, Nih L, Margaill I, et al: MFGE8 inhibits inflammasome-induced IL-1beta production and limits postischemic cerebral 
injury. J Clin Invest 2013, 123:1176-1181.

14. Shi X, Cai X, Di W, Li J, Xu X, Zhang A, Qi W, Zhou Z, Fang Y: MFG-E8 Selectively Inhibited AbetaInduced Microglial M1 Polarization via NF-kappaB and PI3K-Akt Pathways. Mol Neurobiol 2017, 54:7777-7788.

15. Li E, Noda M, Doi Y, Parajuli B, Kawanokuchi J, Sonobe Y, Takeuchi H, Mizuno T, Suzumura A: The neuroprotective effects of milk fat globule-EGF factor 8 against oligomeric amyloid beta toxicity. $J$ Neuroinflammation 2012, 9:148.

16. Soki FN, Koh AJ, Jones JD, Kim YW, Dai J, Keller ET, Pienta KJ, Atabai K, Roca H, McCauley LK: Polarization of prostate cancer-associated macrophages is induced by milk fat globule-EGF factor 8 (MFG-E8)-mediated efferocytosis. J Biol Chem 2014, 289:24560-24572.

17. Gao YY, Zhang ZH, Zhuang Z, Lu Y, Wu LY, Ye ZN, Zhang XS, Chen CL, Li W, Hang CH: Recombinant milk fat globule-EGF factor-8 reduces apoptosis via integrin beta3/FAK/PI3K/AKT signaling pathway in rats after traumatic brain injury. Cell Death Dis 2018, 9:845.

18. Lu Y, Zhang XS, Zhou XM, Gao YY, Chen CL, Liu JP, Ye ZN, Zhang ZH, Wu LY, Li W, Hang CH: Peroxiredoxin 1/2 protects brain against H2O2-induced apoptosis after subarachnoid hemorrhage. FASEB J 2019, 33:3051-3062.

19. Liu F, Hu Q, Li B, Manaenko A, Chen Y, Tang J, Guo Z, Tang J, Zhang JH: Recombinant milk fat globule-EGF factor-8 reduces oxidative stress via integrin beta3/nuclear factor erythroid 2-related factor 2/heme oxygenase pathway in subarachnoid hemorrhage rats. Stroke 2014, 45:3691-3697.

20. Wu L-Y, Ye Z-N, Zhou C-H, Wang C-X, Xie G-B, Zhang X-S, Gao Y-Y, Zhang Z-H, Zhou M-L, Zhuang Z, et al: Roles of Pannexin-1 Channels in Inflammatory Response through the TLRs/NF-Kappa B Signaling Pathway Following Experimental Subarachnoid Hemorrhage in Rats. Frontiers in Molecular Neuroscience 2017, 10.

21. Lu Y, Zhang XS, Zhang ZH, Zhou XM, Gao YY, Liu GJ, Wang H, Wu LY, Li W, Hang CH: Peroxiredoxin 2 activates microglia by interacting with Toll-like receptor 4 after subarachnoid hemorrhage. $J$ Neuroinflammation 2018, 15:87.

22. Zhou C, Xie G, Wang C, Zhang Z, Chen Q, Zhang L, Wu L, Wei Y, Ding H, Hang C, et al: Decreased progranulin levels in patients and rats with subarachnoid hemorrhage: a potential role in inhibiting inflammation by suppressing neutrophil recruitment. J Neuroinflammation 2015, 12:200.

23. Gao Y, Zhuang Z, Gao S, Li X, Zhang Z, Ye Z, Li L, Tang C, Zhou M, Han X, Li J: Tetrahydrocurcumin reduces oxidative stress-induced apoptosis via the mitochondrial apoptotic pathway by modulating autophagy in rats after traumatic brain injury. Am J Transl Res 2017, 9:887-899.

24. Sugawara T, Ayer R, Jadhav V, Zhang JH: A new grading system evaluating bleeding scale in filament perforation subarachnoid hemorrhage rat model. J Neurosci Methods 2008, 167:327-334.

25. Xie Z, Enkhjargal B, Wu L, Zhou K, Sun C, Hu X, Gospodarev V, Tang J, You C, Zhang JH: Exendin-4 attenuates neuronal death via GLP-1R/PI3K/Akt pathway in early brain injury after subarachnoid hemorrhage in rats. Neuropharmacology 2018, 128:142-151. 
26. Gao Y, Li J, Wu L, Zhou C, Wang Q, Li X, Zhou M, Wang H: Tetrahydrocurcumin provides neuroprotection in rats after traumatic brain injury: autophagy and the PI3K/AKT pathways as a potential mechanism. Journal of Surgical Research 2016, 206:67-76.

27. Cahill J, Zhang JH: Subarachnoid hemorrhage: is it time for a new direction? Stroke 2009, 40:S86-87.

28. Yi Y-S: Functional Role of Milk Fat Globule-Epidermal Growth Factor VIII in Macrophage-Mediated Inflammatory Responses and Inflammatory/Autoimmune Diseases. Mediators of Inflammation 2016, 2016:1-12.

29. Brissette MJ, Lepage S, Lamonde AS, Sirois I, Groleau J, Laurin LP, Cailhier JF: MFG-E8 released by apoptotic endothelial cells triggers anti-inflammatory macrophage reprogramming. PLoS One 2012, 7:e36368.

30. Shukla V, Shakya AK, Perez-Pinzon MA, Dave KR: Cerebral ischemic damage in diabetes: an inflammatory perspective. J Neuroinflammation 2017, 14:21.

31. Kojic M, Gaik M, Kiska B, Salerno-Kochan A, Hunt S, Tedoldi A, Mureev S, Jones A, Whittle B, Genovesi $L A$, et al: Elongator mutation in mice induces neurodegeneration and ataxia-like behavior. $\mathrm{Nat}$ Commun 2018, 9:3195.

32. Sun Q, Wu W, Hu YC, Li H, Zhang D, Li S, Li W, Li WD, Ma B, Zhu JH, et al: Early release of highmobility group box 1 (HMGB1) from neurons in experimental subarachnoid hemorrhage in vivo and in vitro. J Neuroinflammation 2014, 11:106.

33. Pan J, Jin JL, Ge HM, Yin KL, Chen X, Han LJ, Chen Y, Qian L, Li XX, Xu Y: Malibatol A regulates microglia M1/M2 polarization in experimental stroke in a PPARgamma-dependent manner. $J$ Neuroinflammation 2015, 12:51.

34. d'Avila JC, Lam TI, Bingham D, Shi J, Won SJ, Kauppinen TM, Massa S, Liu J, Swanson RA: Microglial activation induced by brain trauma is suppressed by post-injury treatment with a PARP inhibitor. J Neuroinflammation 2012, 9:31.

35. Aziz M, Jacob A, Matsuda A, Wang P: Review: milk fat globule-EGF factor 8 expression, function and plausible signal transduction in resolving inflammation. Apoptosis 2011, 16:1077-1086.

36. Aziz M, Jacob A, Matsuda A, Wu R, Zhou M, Dong W, Yang WL, Wang P: Pre-treatment of recombinant mouse MFG-E8 downregulates LPS-induced TNF-alpha production in macrophages via STAT3-mediated SOCS3 activation. PLoS One 2011, 6:e27685.

37. Liang Z, Wu G, Fan C, Xu J, Jiang S, Yan X, Di S, Ma Z, Hu W, Yang Y: The emerging role of signal transducer and activator of transcription 3 in cerebral ischemic and hemorrhagic stroke. Prog Neurobio/ 2016, 137:1-16.

38. Sehara Y, Sawicka K, Hwang JY, Latuszek-Barrantes A, Etgen AM, Zukin RS: Survivin Is a transcriptional target of STAT3 critical to estradiol neuroprotection in global ischemia. $J$ Neurosci 2013, 33:12364-12374.

39. Giancotti FG, Ruoslahti E: Integrin signaling. Science 1999, 285:1028-1032.

40. Behera R, Kumar V, Lohite K, Karnik S, Kundu GC: Activation of JAK2/STAT3 signaling by osteopontin promotes tumor growth in human breast cancer cells. Carcinogenesis 2010, 31:192-200. 


\section{Supplementary Figure Caption}

Figure S1. Experimental designs and animal groups. WB: western blot; rt-PCR: quantitative real-time polymerase chain reaction; IF: immunofluorescence; h: hour; d: days; rh-MFG-E8: recombinant human milk fat globule-EGF factor-8; i.c.v: intracerebroventricular; TUNEL: terminal deoxynucleotidyl transferase dUTP nick endlabeling staining; FJC: Fluoro-Jade C; Stattic: p-STAT3 inhibitor;

\section{Figures}
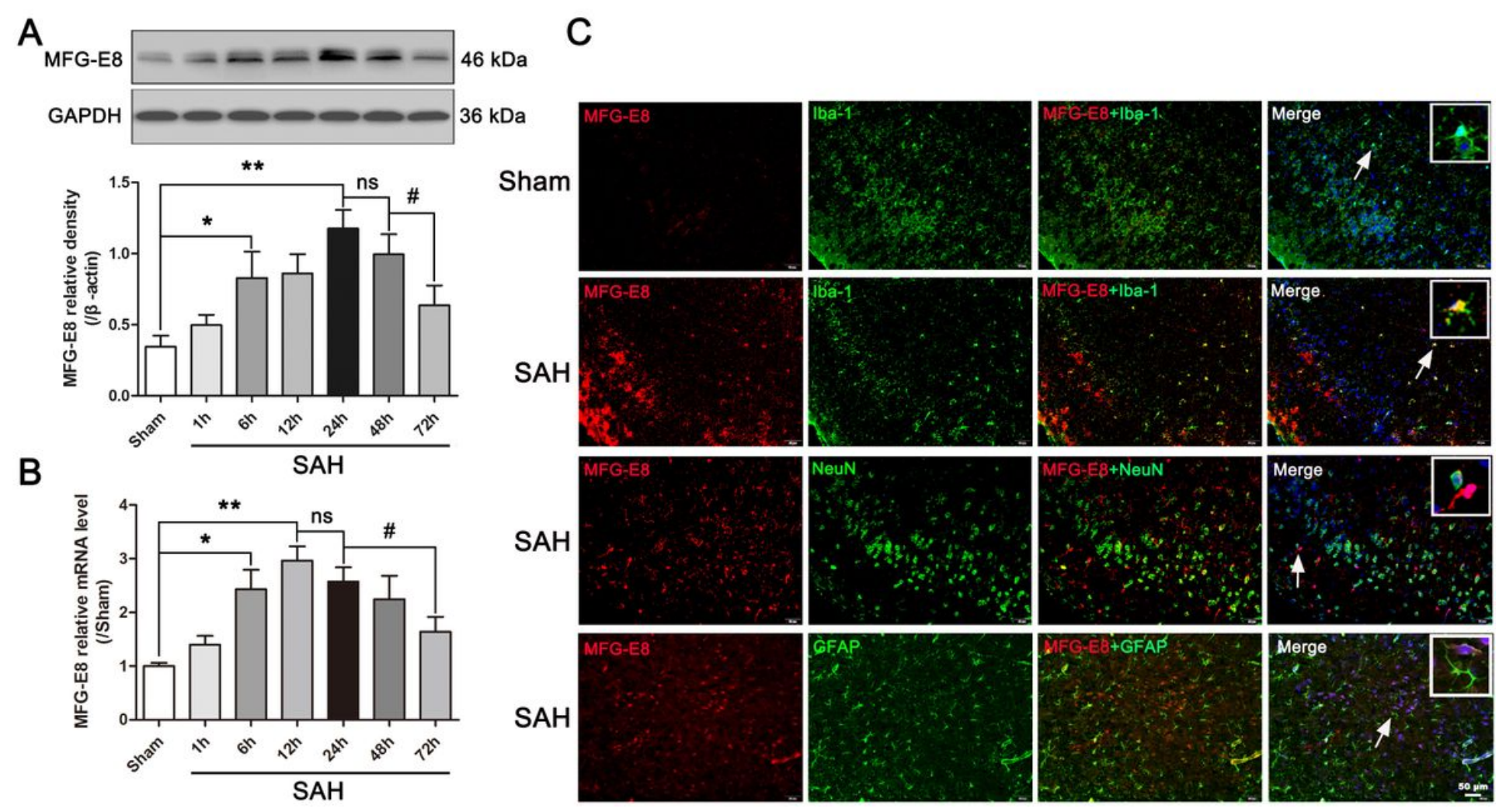

Figure 1

Endogenous expression of MFG-E8 on the mRNA and protein levels, and cellular distribution after SAH. (a) Western blotting showed that the protein level of MFG-E8 increased after SAH and peaked at 24 as compared with sham group. (b) The mRNA level of MFG-E8 increased dramatically and peaked at $12 \mathrm{~h}$ after SAH. (c) Representative immunofluorescence staining images of MFG-E8/Iba-1, MFG-E8/NeuN, MFG-E8/GFAP in the Sham group and SAH group at $24 \mathrm{~h}$. MFG-E8 mainly expressed in Iba- $1+$ microglia after $\mathrm{SAH}$, and also some located in neurons, but not in astrocytes. Arrow point to the magnified area in the top right corner. The quantitative data are the mean $\pm S D\left(n=5\right.$, each; ${ }^{*} \otimes 0.05 ;{ }^{\star \star} p \otimes 0.01 \mathrm{vs}$. Sham group; $\# \mathrm{p} \otimes 0.05$ vs. SAH (24 h) group, SAH (48 h) group; nsp $\otimes 0.05)$. Scale bars $=50 \mu \mathrm{m}$. 


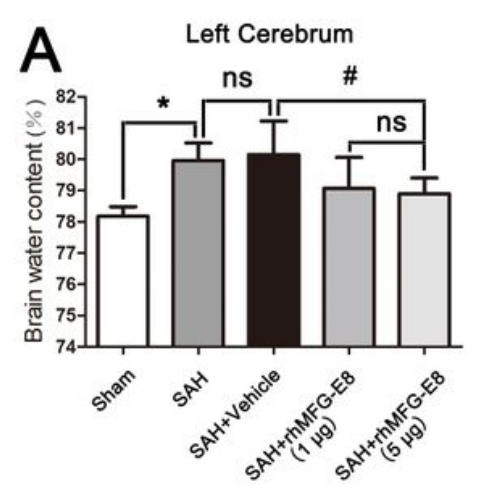

E Sham SAH

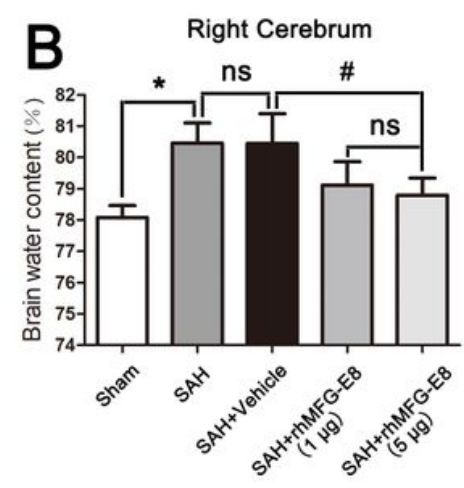

SAH+Vehicle
C

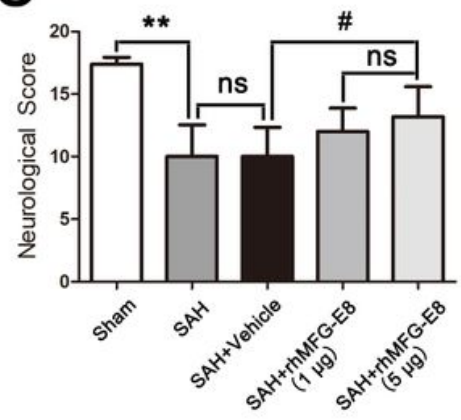

D

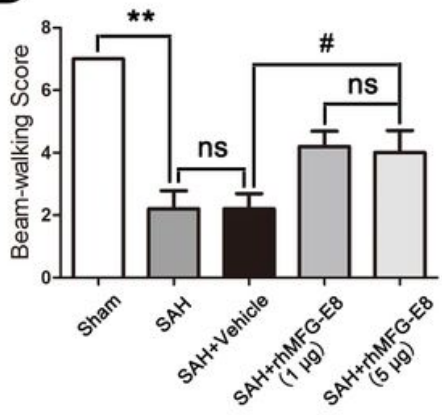

$\mathbf{F}$

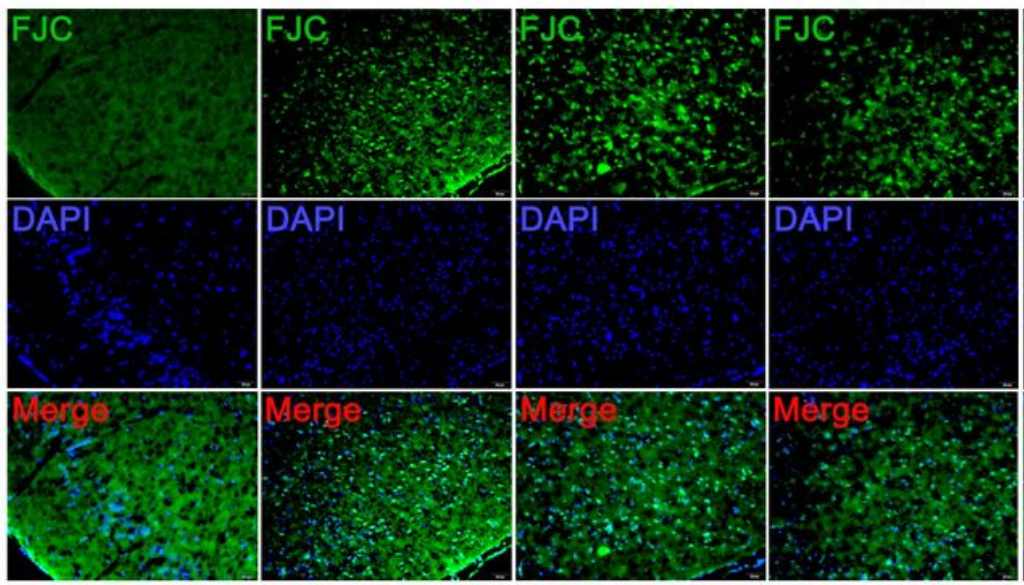

SAH+rhMFG-E8 $(5 \mu \mathrm{g})$
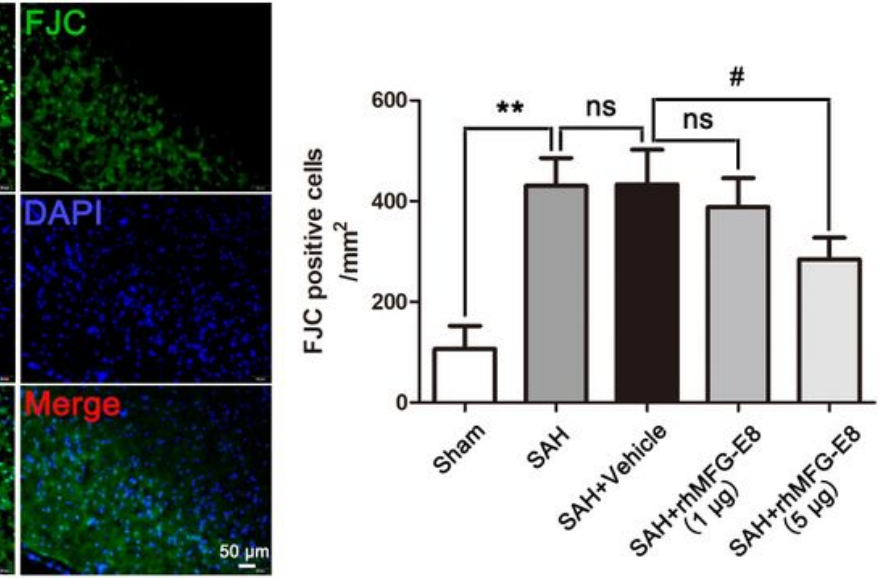

\section{Figure 2}

Effects of rhMFG-E8 on neuroprotection were evaluated by brain water content, neurological severity scores, beam-walking score and Fluoro Jade $\mathrm{C}$ staining (FJC) at $24 \mathrm{~h}$ after SAH. High dosage of rhMFGE8 significantly decreased brain edema $(a, b)$, improved neurological function (c, d) at $24 \mathrm{~h}$ after SAH. Representative immunofluorescence staining images of FJC (green) (e). High dosage of rhMFG-E8 remarkably decreased the amount of FJC-positive cells after SAH. The quantitative data are the mean \pm $S D\left(n=5\right.$, each; ${ }^{\star} p \otimes 0.05 ;{ }^{* \star} p \otimes 0.01$ vs. Sham group; $\# p \otimes 0.05$ vs. SAH + Vehicle group; $\left.n s p \otimes 0.05\right)$. Scale bars $=50 \mu \mathrm{m}$. 


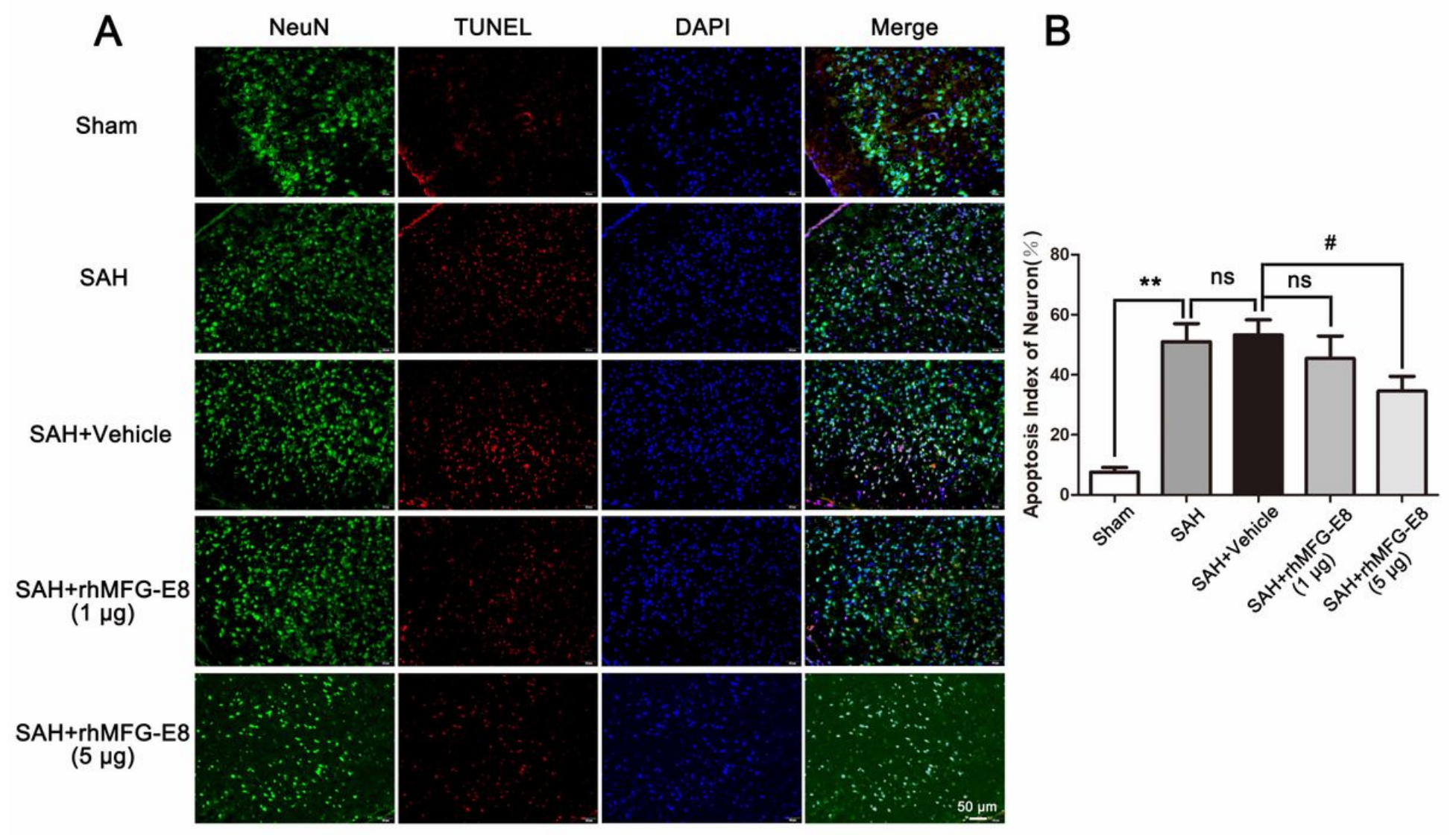

Figure 3

Effect of rhMFG-E8 treatment on neuronal apoptosis at $24 \mathrm{~h}$ after SAH. (a) Representative immunofluorescence staining images of TUNEL (NeuN = green, TUNEL = red, DAPI = blue). (b) Quantitative analysis of TUNEL positive cells showed that high dosage of rhMFG-E8 significantly decreased the amount of TUNEL-positive cells at $24 \mathrm{~h}$ after SAH. The quantitative data are the mean \pm SD $\left(n=3\right.$, each; ${ }^{* \star p} \otimes 0.01$ vs. Sham group; $\# p \otimes 0.05$ vs. SAH + Vehicle group; $\left.n s p \otimes 0.05\right)$. Scale bars $=50$ $\mu \mathrm{m}$. 
A

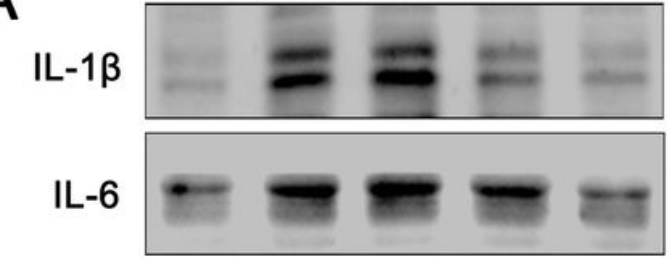

TNF- $\alpha$

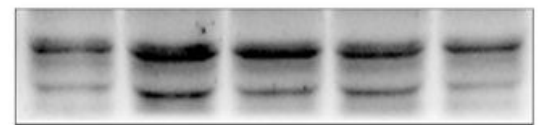

IL-10

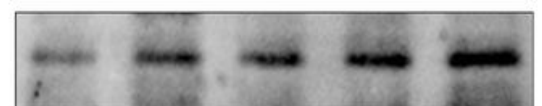

$\beta$-actin

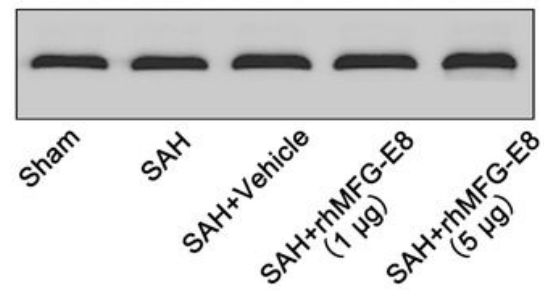

$17 \mathrm{kDa}$

$21 \mathrm{kDa}$

$16 \mathrm{kDa}$

20 kDa

$43 \mathrm{kDa}$

D
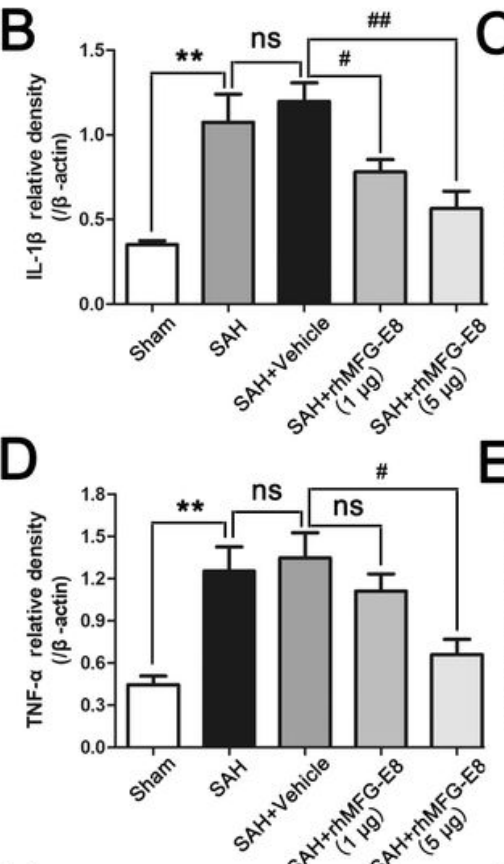

$\mathrm{H}$

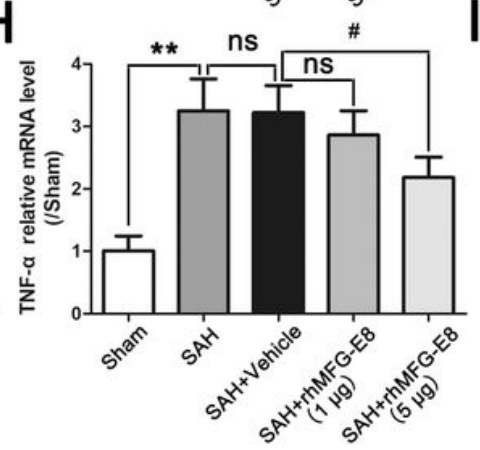

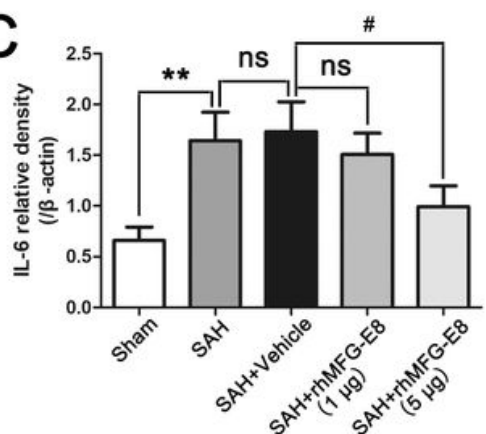

E
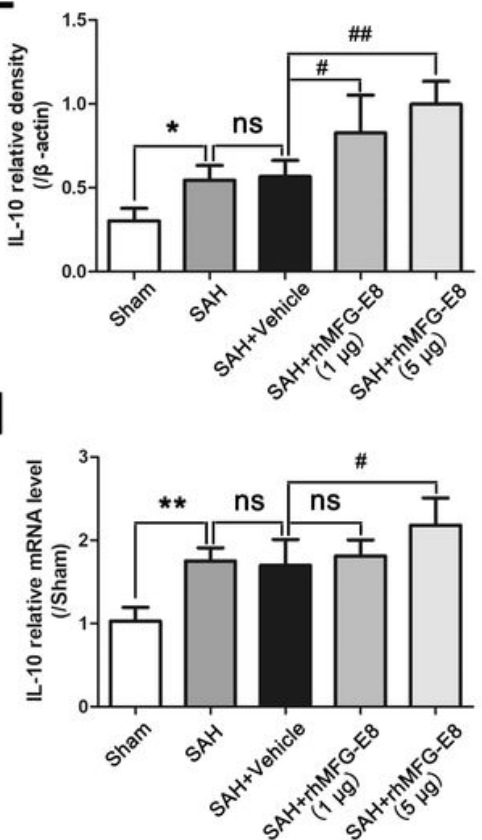

Figure 4

The effect of rhMFG-E8 on neuron-inflammation at $24 \mathrm{~h}$ after SAH. (a) Western blotting showed that administration of high dosage rhMFG-E8 obviously reduced the expressions of pro-inflammation mediators IL-1 $\beta$ (b), IL-6 (c), TNF-a (d) and increased the level of anti-inflammation factor IL-10 (e) at $24 \mathrm{~h}$ after SAH. The mRNA of pro-inflammation cytokines IL-1 $\beta$ (f), IL-6 (g), TNF-a (h) in SAH group were expressed as fold change to sham group, and decreased after rhMFG-E8 $(5 \mu \mathrm{g})$ treatment, meanwhile increased the mRNA level of IL-10 (I). The quantitative data are the mean $\pm S D\left(n=5\right.$, each; ${ }^{\star} p \otimes 0.05$, **p $\otimes 0.01$ vs. Sham group; $\# p \otimes 0.05, \# \# p \otimes 0.01$ vs. SAH + Vehicle group; $n s p \otimes 0.05)$. 

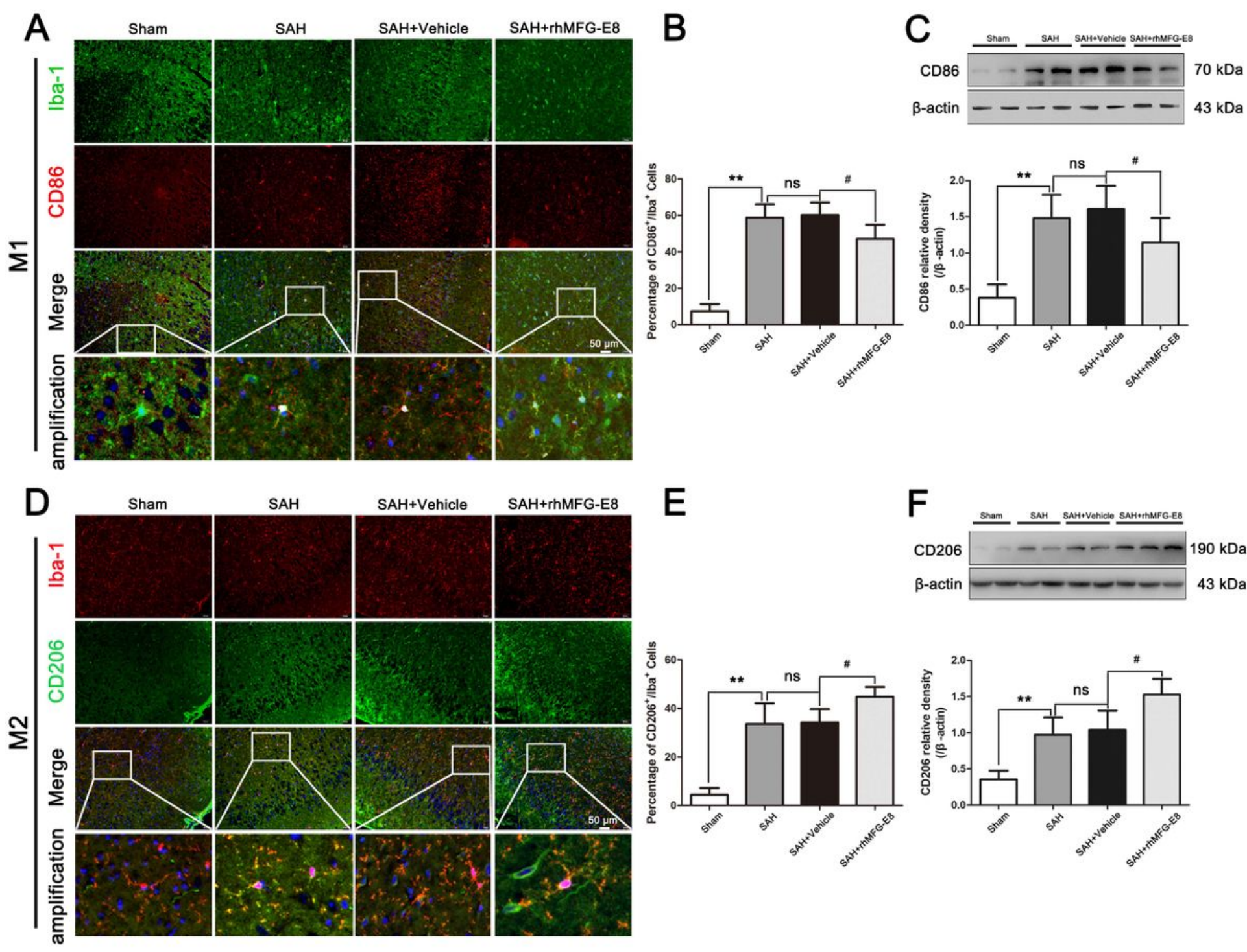

\section{Figure 5}

The effect of rhMFG-E8 on microglia phenotypic transformation at $24 \mathrm{~h}$ after SAH. Representative immunofluorescence staining images of M1 phenotype (a) and M2 phenotype (d), and its quantification analysis (b, e), which showed that the proportion of M1 microglia (Iba-1+/CD86+) and M2 (Iba$1+/$ CD206+) were increased after SAH as compared with sham group, while treatment with rhMFG-E8 (5 $\mu \mathrm{g})$ decreased the amount of M1 and further increased the quantity of M2 microglia. Western blotting showed that SAH induction increased the expressions of M1 marker CD86 (c) and M2 marker CD206 (f) when compared with sham group. Administration of rhMFG-E8 $(5 \mu \mathrm{g})$ decreased the protein level of CD86, while increased the expression of CD206 relative to the SAH + Vehicle group. The quantitative data are the mean $\pm S D(n=5$, each; $* * p \otimes 0.01$ vs. Sham group; \#p $\otimes 0.05$ vs. $S A H+V e h i c l e ~ g r o u p ; ~ n s p ~ \otimes 0.05)$. 

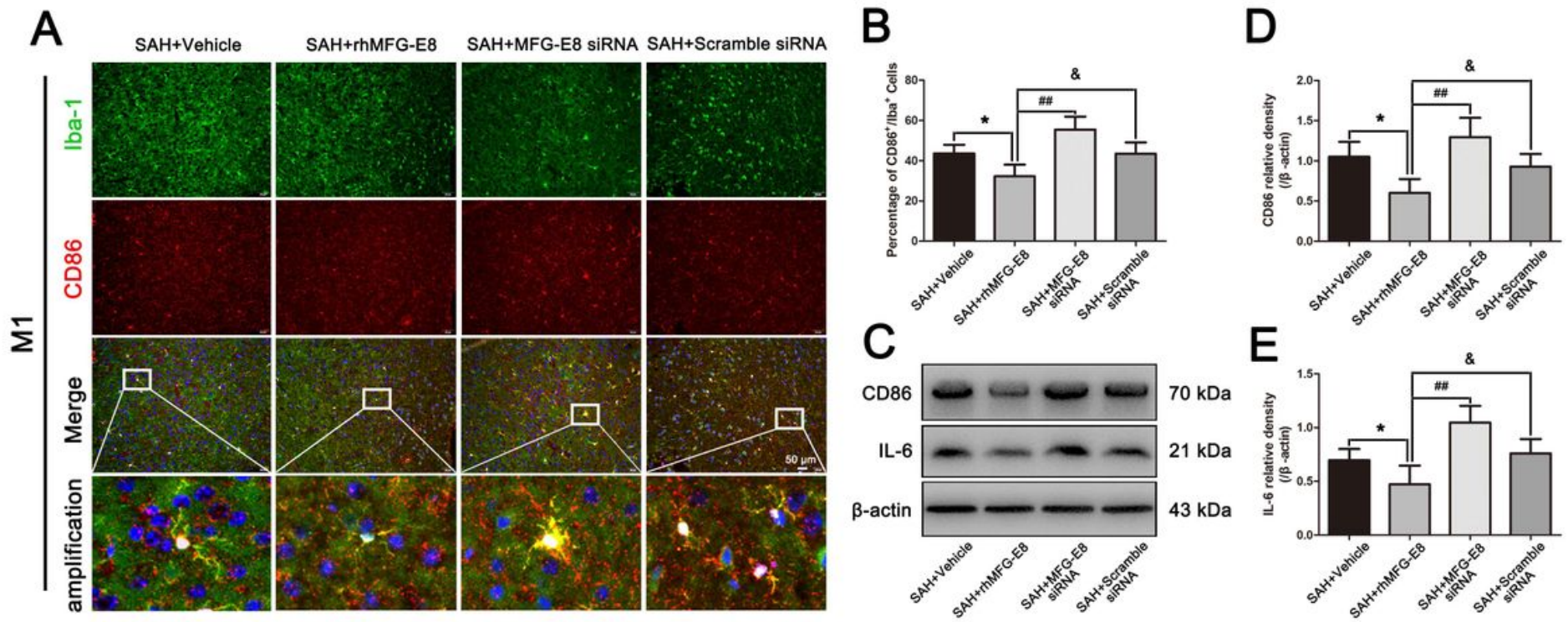

$\mathrm{F}$

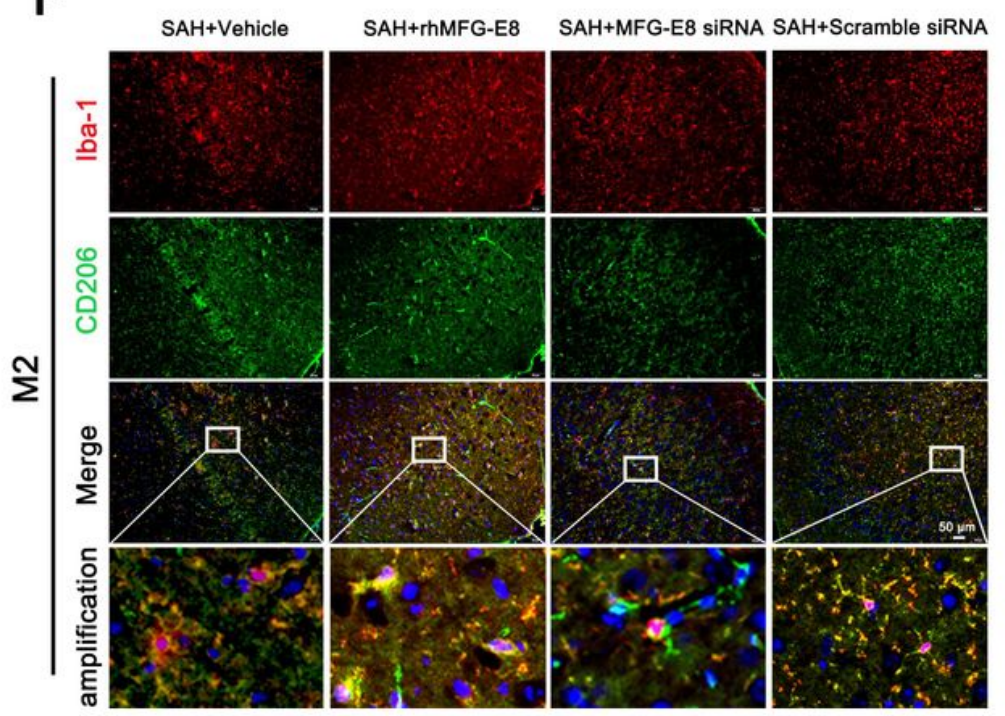

G
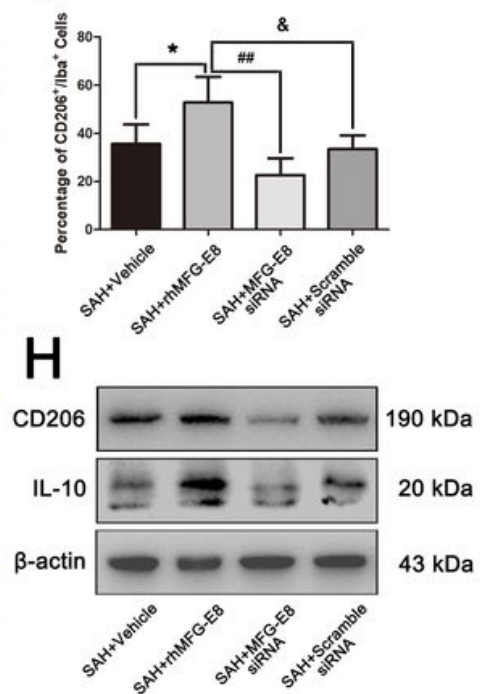

I
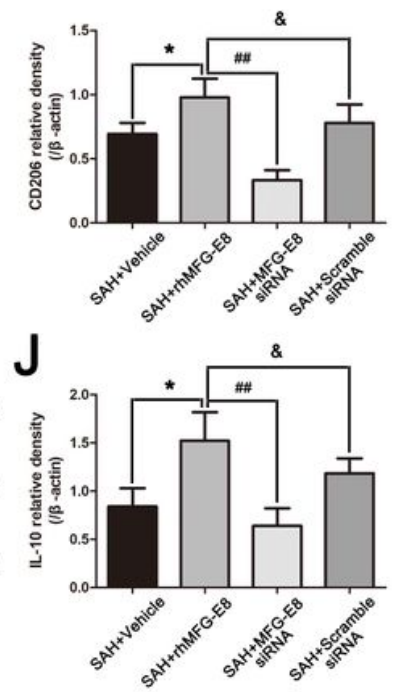

Figure 6

Silencing MFG-E8 aggravated neuro-inflammation and microglia transformed into M1 phenotype.

Representative immunofluorescence staining images of M1 phenotype (a) and M2 phenotype (f), showed that silencing MFG-E8 by siRNA abolished the effect of MFG-E8 on M2 phenotype switch compared with the SAH + rhMFG-E8 group (b, g). Meanwhile, Western blotting $(c, h)$ showed that treatment with MFG-E8 siRNA increased the protein levels of CD86 (d) and IL-6 (e), and decreased the expression of CD206 (i) and IL-10 (j) when compared with the SAH + rhMFG-E8 group. The quantitative data are the mean \pm SD ( $n$ $=5$, each; ${ }^{* *} \mathrm{p} \otimes 0.01$ vs. SAH + Vehicle group; \#\#p $\otimes 0.01$ vs. SAH + rhMFG-E8 group; \&p $\otimes 0.05$ vs. SAH + rhMFG-E8 group). 


\section{SAH}

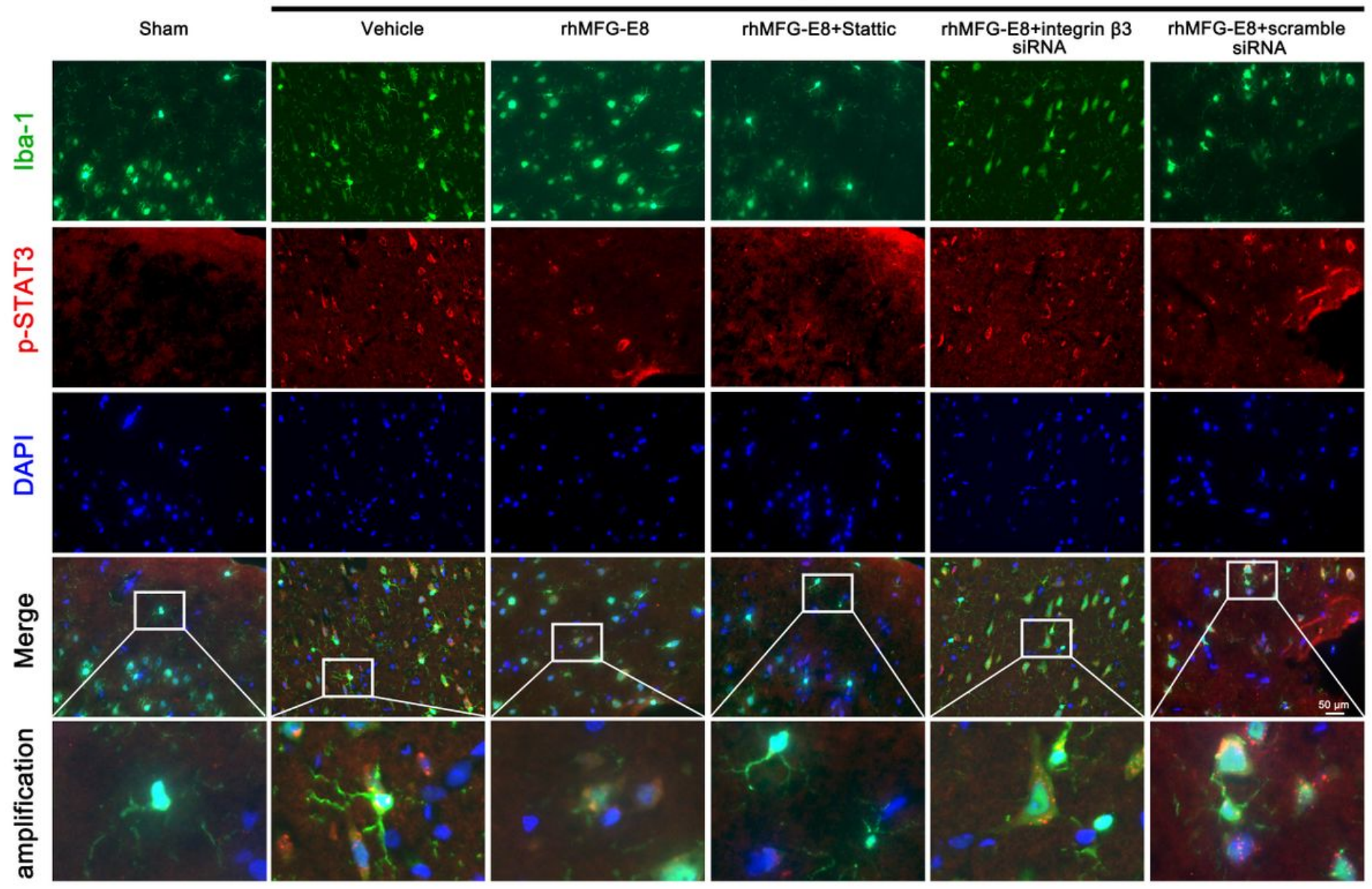

\section{Figure 7}

Integrin $\beta 3$ siRNA and Stattic pretreatment abolished the effect of rhMFG-E8 on p-STAT3 at $24 \mathrm{~h}$ after SAH. Representative immunofluorescence staining images of p-STAT3 (red) in microglia (Iba-1, green), exhibited that p-STAT3 punctate dots were noticeably observed in cytoplasm, and some translocated into nucleus after SAH, compared with SAH + rhMFG-E8 group and SAH + rhMFG-E8 + Stattic group. While treatment of integrin $\beta 3$ siRNA, amounts of p-STAT3 punctate dots in cytoplasm and nucleus of microglia were observed, compared to the SAH +rhMFG-E8 group and SAH + rhMFG-E8 + Stattic group. 

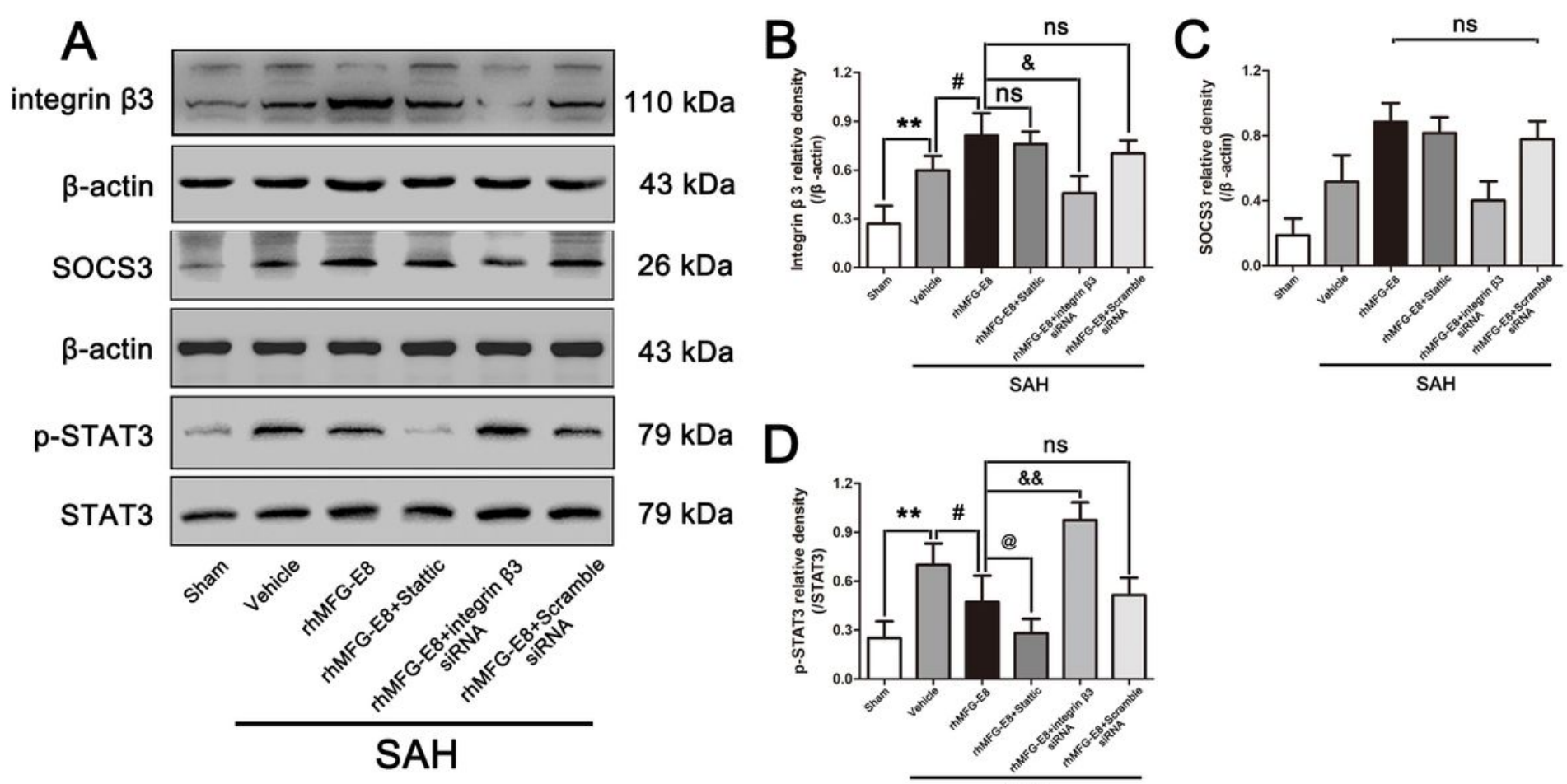

79 kDa D
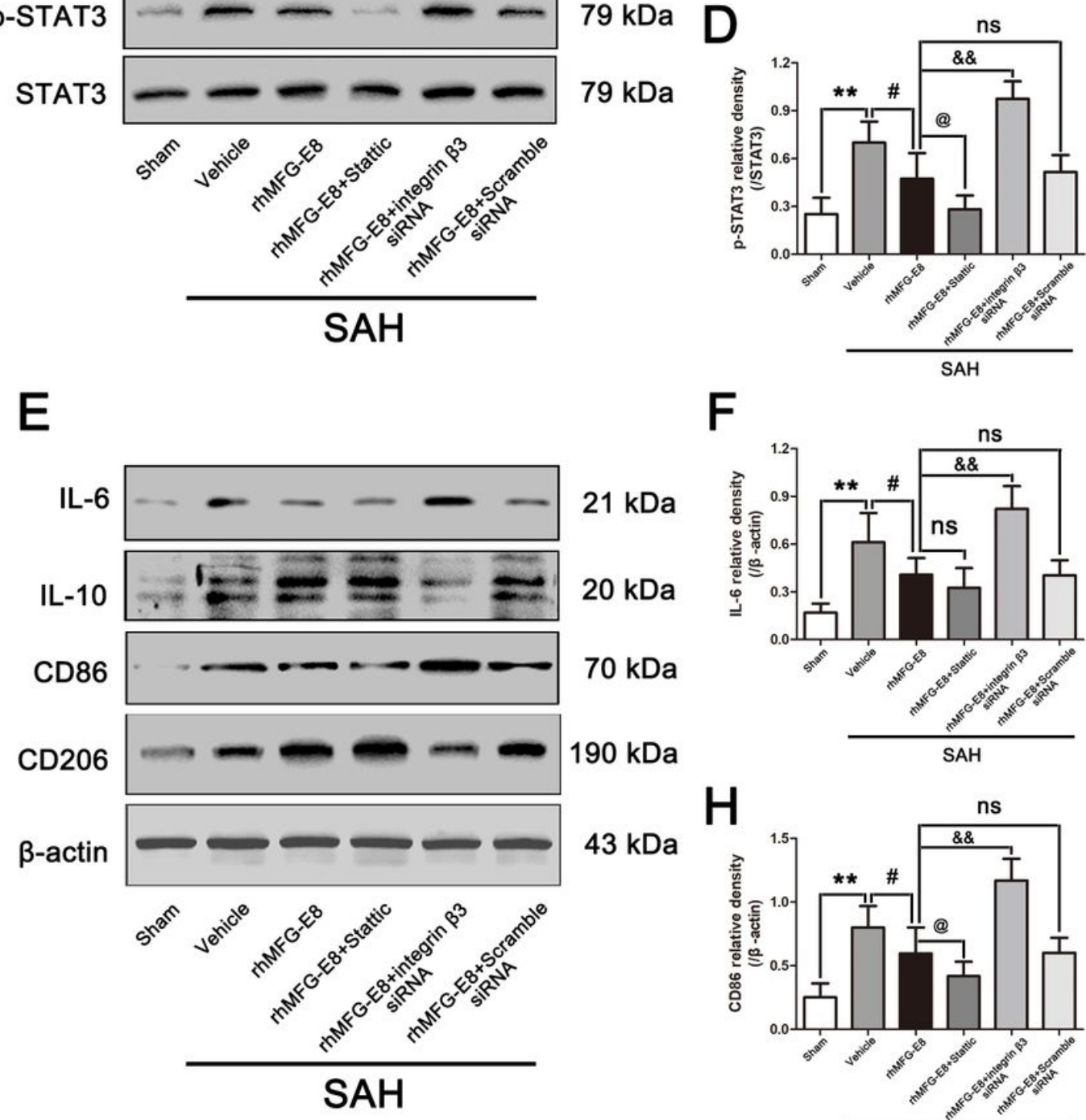

G

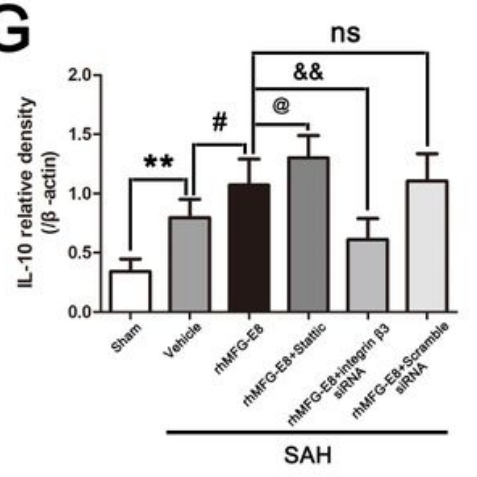

$43 \mathrm{kDa}$
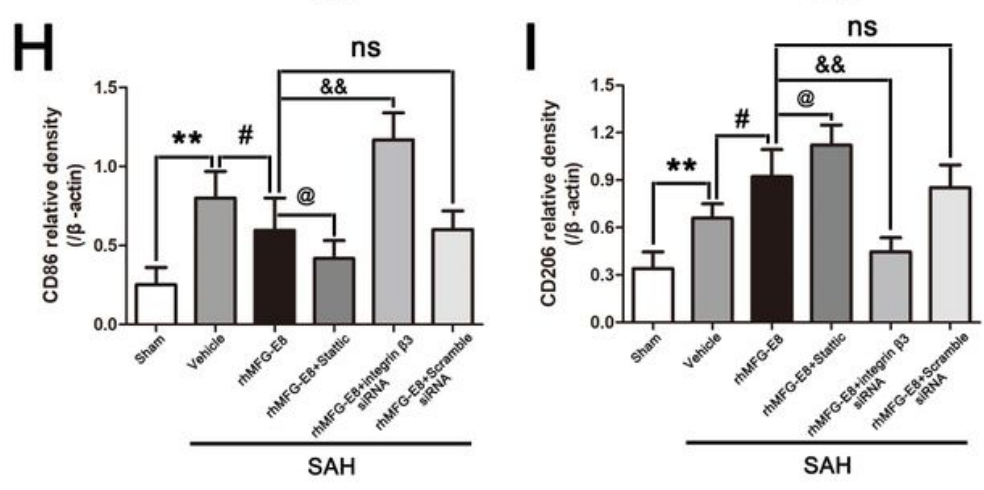

Figure 8

The role of integrin $33 /$ SOCS3/STAT3 pathway on rhMFG-E8 of microglial polarization at $24 \mathrm{~h}$ after SAH. Western blotting $(a, e)$ showed that treatment with integrin $\beta 3$ siRNA decreased the expressions of SOCS3 (c) and M2 markers (IL-10, CD206) (g, i), and increased the protein levels of p-STAT3 (d) and M1 markers $(\mathrm{IL}-6, \mathrm{CD} 86)(\mathrm{f}, \mathrm{h})$ compared with SAH + rhMFG-E8 group. Administration of STAT3 inhibitor, Stattic (5 $\mu \mathrm{M}$ ) only decreased the expression of M1 markers (IL-6, CD86) $(f, h)$, and increased the M2 markers (IL10, CD206) (g, i) compared to SAH + rhMFG-E8 group, while had no effect on the levels of integrin $\beta 3$ (b) 


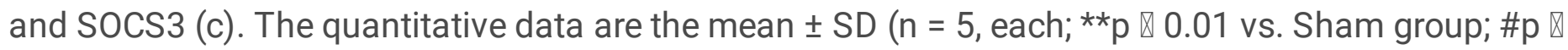
0.05 vs. $S A H+$ Vehicle group; \&p $\otimes 0.05$ vs. SAH + rhMFG-E8 group; \&p $\otimes 0.05, \& \& p \otimes 0.01$ vs. $S A H+$ rhMFG-E8 group; nsp $\otimes 0.05)$.

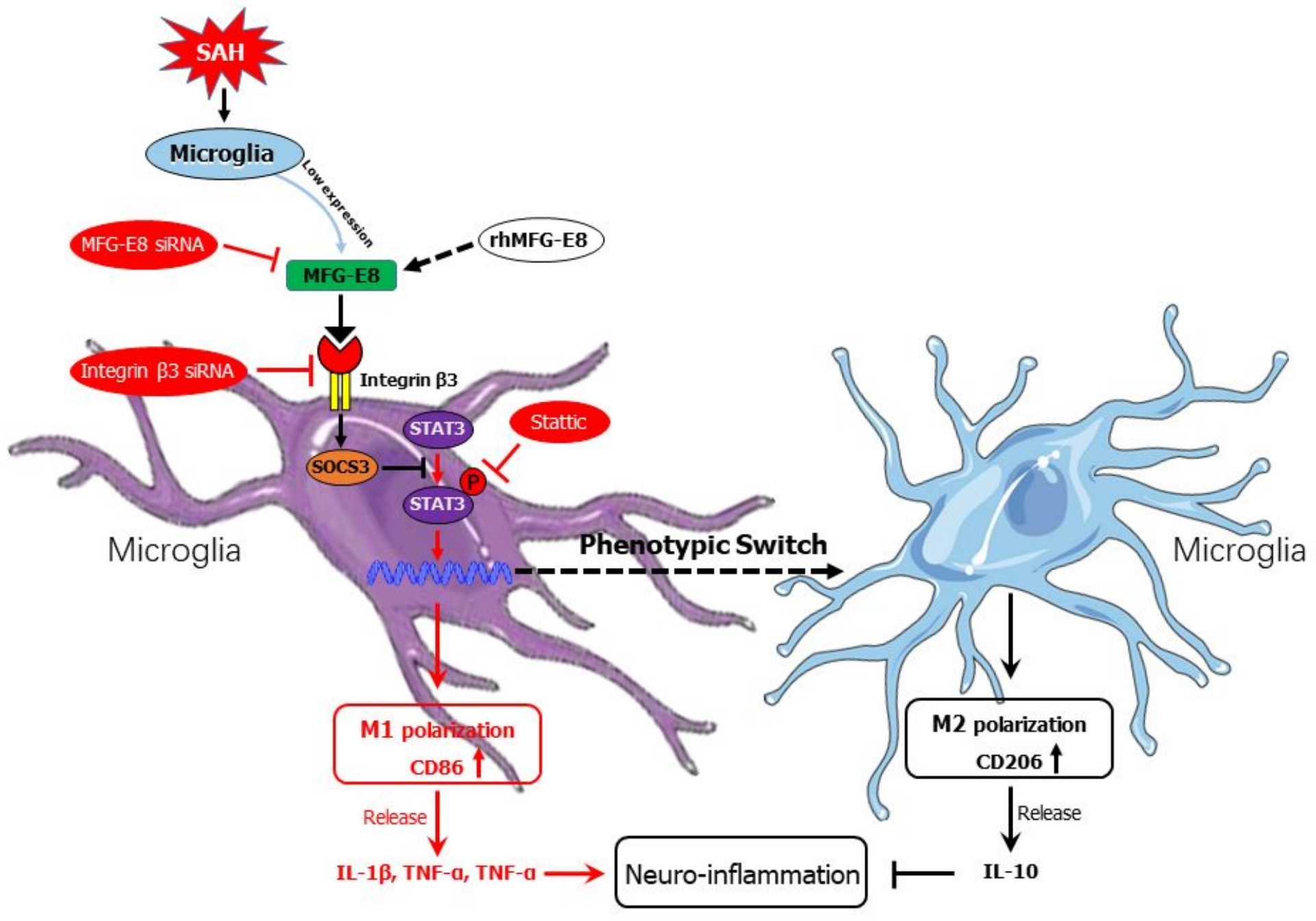

\section{Figure 9}

Proposed model of MFG-E8 mediated neuro-inflammation and microglial polarization through integrin B3/SOCS3/STAT3 pathway after SAH. SAH induction resulted in up-regulated endogenous expression of MFG-E8 in microglia, while remain at low level, then activated phosphorylation of STAT3, lead to M1 polarization and aggravated neuro-inflammation injury. Treatment with rhMFG-E8 increased the protein levels of integrin $\beta 3$ receptor and SOCS3, the latter as a negative regulator of STAT3, to activate microglia and promote microglial phenotype transformed into $\mathrm{M} 2$, and released anti-inflammation cytokine (IL-10) to alleviate inflammation damage. Moreover, inhibition of MFG-E8 via siRNA and p-STAT3 by Stattic to further verified that MFG-E8 modulated neuro-inflammation and microglial polarization might through regulating integrin $\beta 3 / \mathrm{SOCS} 3 / \mathrm{STAT} 3$ signaling pathway after SAH.

\section{Supplementary Files}


This is a list of supplementary files associated with this preprint. Click to download.

- AdditionalfileTable3.docx

- AdditionalfileTable1.docx

- AdditionalfileTable2.doc

- FigureS1.jpg 